\title{
How (Not) to Integrate Blood Subtyping Technology to Kidney Exchange*
}

\author{
Tayfun Sönmez $\quad$ M. Utku Ünver ${ }^{\ddagger} \quad$ Özgür Yılmaz
}

October 2017

\begin{abstract}
Even though kidney exchange became an important source of kidney transplants over the last decade with the introduction of market design techniques to organ transplantation, the shortage of kidneys for transplantation is greater than ever. Due to biological disadvantages, patient populations of blood types $\mathrm{B} / \mathrm{O}$ are disproportionately hurt by this increasing shortage. The disadvantaged blood types are overrepresented among minorities in the US. In order to mitigate the disproportionate harm to these biologically disadvantaged groups, the UNOS reformed in 2014 the US deceased-donor kidney-allocation system, utilizing a technological advance in blood typing. The improved technology allows a certain fraction of blood type A kidneys, referred to as subtype A2 kidneys, to be transplanted to medically qualified patients of blood types B/O. The recent reform prioritizes subtype A2 deceased-donor kidneys for blood type B patients only. When restricted to the deceased-donor allocation system, this is merely a distributional reform with no adverse impact on the overall welfare of the patient population. In this paper we show that the current implementation of the reform has an unintended consequence, and it de facto extends the preferential allocation to kidney exchange as well. Ironically this "spillover" not only reduces the number of living-donor transplants for the overall patient population, but also for the biologically disadvantaged groups who are the intended beneficiaries of
\end{abstract}

*Sönmez acknowledges the research support of Goldman Sachs Gives via the Dalinc Ariburnu - Goldman Sachs Faculty Research Fund. Sönmez and Ünver acknowledge the research support of NSF via award SES \#1426440. Yılmaz acknowledges the research support of TÜBİTAK via program 2219.

${ }^{\dagger}$ Department of Economics, Boston College, and Distinguished Research Fellow, Koç University; sonmezt@bc.edu; www.tayfunsonmez.net

${ }_{\ddagger}^{\ddagger}$ Department of Economics, Boston College, and Distinguished Research Fellow, Koç University; unver@bc.edu; www2.bc.edu/utku-unver

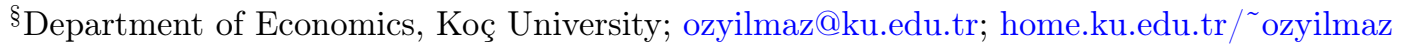


the reform. We show that minor variations of the current policy do not suffer from this unintended consequence, and we make two easy-to-implement, welfare-increasing policy recommendations.

Keywords: Market design, matching, kidney exchange

JEL Classification Numbers: C71, C78, D02, D47, D63, I10

\section{Introduction}

Following a series of collaborations between members of the transplantation community and researchers in market design, kidney exchange became an important source of transplant kidneys over the last decade (cf. Roth, Sönmez, and Ünver, 2004, 2005b, 2007). By 2010, transplants from kidney exchanges in the US exceeded 550 (Massie et al., 2013), which account for about 10 percent of all living-donor kidney transplantations and more than 3 percent of all kidney transplantations. Given the persistent shortage of kidneys for transplantation throughout the world, analysis of policies and procedures that could increase the contribution of kidney exchange to the number of kidney transplants has become an active area of research not only in the transplantation community, but also among the researchers in market design. In this paper we analyze the potential spillovers of a recent policy change in the allocation of deceaseddonor kidneys on the number of transplants from living donors, including those from kidney exchanges. We show that explicit or de facto extension of this policy to kidney exchange can result in a reduction of the number of transplants from living donors (due to a reduction of transplants via exchanges), even though a number of the policy's variants offer great promise to increase the number of transplants. In order to explain the 2014 policy change and its potential effect on the number of living-donor transplants, we give some basic background on transplant immunology.

The most widely used blood type classification is known as the ABO grouping. Under this classification, there are two antigens (antigens A, B) and two antibodies (antibodies anti-A, anti-B) that are responsible for the ABO blood types. The specific combination of these four components determines an individual's blood type. For example, people with type A blood have only the A antigen on the surface of their red cells, and, as a result, they do not produce anti-A antibodies (otherwise they would cause the destruction of their own blood.) Thus, only antibody anti-B is produced by blood type A people. Similarly, blood type B people have antigen B along with antibody anti-A, whereas blood type $\mathrm{O}$ people have neither antigen while carrying both antibodies, and blood type AB people have both antigens while carrying neither antibodies. Blood-type compatibility is a major factor for the successful transplantation of kidneys, and this immunologic asymmetry between different blood types, along with the relative scarcity of type 
B kidneys for transplantation, has historically resulted in an unfavorable situation for blood type $\mathrm{O}$ and $\mathrm{B}$ patients. The impact of this imbalance has not been uniform across different ethnicities, and it has especially disadvantaged the African-American patient population, of which nearly 70 percent have blood types $\mathrm{O}$ or $\mathrm{B}$.

The above mentioned ABO blood type induced imbalance is well known and well analyzed. What is less known is that antigen A has two major subtypes, A1 and A2, and different immunologic properties of these subtypes have started to play an important role in the allocation of deceased-donor kidneys in the US since 2014. When donated to type B or O patients, subtype A2 kidneys generate a significantly weaker antibody response than subtype A1 kidneys. ${ }^{1}$ The resulting distribution of antibody response in the patient population is such that A2 kidneys can be safely transplanted to more than 80 percent of type B patients and to approximately 30 percent of type O patients (see for example Bryan, 2014; Nelson et al., 2002). This medical possibility is the basis of a recent policy change in the US to provide greater kidney access to blood type B patients: In 2014, the United Network for Organ Sharing (UNOS) ${ }^{2}$ approved a new national deceased-donor kidney-allocation system that preferentially allocates subtype A2 kidneys to type $\mathrm{B}$ patients. While both type $\mathrm{O}$ and $\mathrm{B}$ patients can potentially receive subtype A2 kidneys, the preferential allocation is limited to only type B patients. This has two major reasons:

1. Equity in Access: One of the primary goals of the Organ Procurement and Transplantation Network $(\mathrm{OPTN})^{3}$ is to increase and ensure the equity of organ sharing in the national system of organ allocation. ${ }^{4}$ As we mentioned above, blood type $\mathrm{B} / \mathrm{O}$ patients have significantly less access to transplant kidneys than blood type A/AB patients. And while both disadvantaged blood types are overrepresented among minorities, a blood type $\mathrm{B}$ patient is more likely to be a minority than a blood type $\mathrm{O}$ patient. ${ }^{5}$ Hence the preferential allocation of subtype A2 deceased-donor kidneys to blood type B pa-

\footnotetext{
${ }^{1}$ In the US approximately 40 percent of the population is of blood type A. Of this 40 percent, about 80 percent is of subtype A1 and 20 percent is of subtype A2. There are also rare subtypes that are immunologically similar to subtype A2. These rare subtypes, combined, account to less than 1 percent of the blood type A population.

${ }^{2}$ The federal contractor, which is in charge of deceased-donor allocation in the US.

${ }^{3}$ The National Organ Transplant Act (NOTA) of 1984 called for an organ procurement and transplantation network to be created and run by a private, non-profit organization under federal contract.

${ }^{4}$ The federal Final Rule of the OPTN, adopted in March 2000 (Human and Health Services, 1998), provides a regulatory framework for the structure and operation of the OPTN: The primary goal of the OPTN is to increase and ensure the effectiveness, efficiency, and equity of organ sharing in the national system of organ allocation, and to increase the supply of donated organs available for transplantation. Then OPTN started to contract UNOS to run the deceased-donor allocation system in the US.

${ }^{5}$ See, for example, Table 1 in Section 5 Distribution $\mathrm{C}$ for blood type distributions for new patients from different races.
} 
tients is a particularly favorable utilization of deceased-donor A2 kidneys for the minority population as a whole, and especially the African-American patient population, which historically had the lowest access to transplant kidneys.

2. Practicality: For a patient to be eligible for a subtype A2 kidney, his antibody anti-A ( $\operatorname{IgG})$ titer value should be consistently below a certain threshold over a period of time. ${ }^{6}$ Based on this medical criteria, more than 80 percent of type B patients are eligible to receive subtype A2 kidneys. In contrast, only 30-40 percent of type $\mathrm{O}$ patients are eligible for subtype A2 kidneys. Hence directing A2 kidneys to B patients requires regular IgG antibody testing for only blood type B patients, who are more likely to be eligible for these kidneys.

Transplants from deceased donors are not the only source of transplant kidneys. Transplants from living donors, directly or via kidney exchanges, are the two other sources. One implication of the preferential allocation of subtype A2 deceased-donor kidneys to blood type B patients is the routine testing of blood type B patients for their titer levels of antibody anti-A. In contrast, antibody anti-A testing is not a routine process for blood type O patients, especially for those who do not have a blood type A donor who may be of subtype A2. This asymmetry between blood type $\mathrm{B}$ and $\mathrm{O}$ patients results in a de facto extension of the preferential allocation of subtype A2 kidneys to type B patients through kidney exchange as well. That is because, unlike type $\mathrm{B}$ patients, type $\mathrm{O}$ patients often lack the established antibody anti-A testing history necessary to receive a subtype A2 kidney. ${ }^{7}$ Critically, this has an important welfare implication for kidney exchange, unlike for deceased-donor allocation where preferential allocation of subtype A2 kidneys to B patients is mostly a distributional matter. In this paper we show that extending the preferential allocation of A2 kidneys to blood type B patients in kidney exchange not only potentially reduces the total number of living-donor kidney transplants, but also potentially reduces the number of living-donor transplants across all ethnicities, including the most disadvantaged groups such as the African-American patient population. In contrast, making subtype A2 kidneys available to patients with type B or O blood unambiguously benefits patients of all ethnicities. This improvement simply requires establishing an antibody

\footnotetext{
${ }^{6} \mathrm{~A}$ threshold of 1:8 shall be maintained in the US for the 6 months before the transplant (for example, see Nelson and Bryan, 2010).

${ }^{7}$ For example, the operational guidelines of the UNOS National Kidney Exchange Pilot Program allow the possibility of A2-to-O transplants (Organ Procurement and Transplantation Network, 2016), and such compatibility is treated no differently than A2/A2B-to-B transplants in exchanges, unlike in the new UNOS deceased-donor allocation policy. However, because extended $\mathrm{O}$ patient anti-A antibody titer testing is not practiced and such histories are not available as noted above, the national program's policy is de facto A2-to-B compatibility.
} 
anti-A titer level history for blood type $\mathrm{O}$ patients as in the case of blood type $\mathrm{B}$ patients who already have their levels tested to potentially benefit from the preferential allocation of subtype A2 kidneys under the national deceased-donor kidney-allocation system.

A further increase in the number of transplants is possible through a second proposal. We also propose the subtyping tests for the donors to be conducted only after blood/tissuetype-incompatible pairs join a kidney-exchange pool. A practical way to make this proposal applicable requires the help of the health-care systems in the US and elsewhere. We propose the sophisticated, expensive subtyping tests are paid by health insurance companies with the condition that blood type A subtype compatible pairs will be nudged to participate in direct donation only if they cannot be matched through exchange.

Increasingly, economists are taking advantage of advances in technology to design new or improved allocation mechanisms in practical applications including different aspects of kidney exchange. After the initial papers of Roth, Sönmez, and Ünver (2004, 2005b), Roth, Sönmez, and Ünver (2007) quantify the welfare gains associated with allowing larger exchange cycles and show that in a sufficiently large kidney exchange pool, most gains from exchanges can be exhausted by 2\&3-way exchanges. Roth, Sönmez, and Ünver (2005c); Ashlagi and Roth (2014); Toulis and Parkes (2015); Sönmez and Ünver (2015) study whether multiple kidney exchanges can be consolidated into one large one to create a thick market; Roth, Sönmez, and Ünver (2005a); Sönmez and Ünver (2014); Nicolò and Rodriguez-Álvarez (2017); Sönmez, Ünver, and Yenmez (2017) study the welfare gains associated with including compatible pairs into kidney exchange in Pareto sense; Roth et al. (2006); Rees et al. (2009); Ashlagi et al. (2012); Anderson et al. (2017) introduce, present applications, and analyze the effects and welfare gains associated with altruistic-donor-initiated exchange chains. The effects of elimination of immunological incompatibilities in the kidney exchange framework through immunosuppressants were studied by Andersson and Kratz (2016) for blood-type compatibility and by Chun, Heo, and Hong (2016) for both tissue- and blood-type incompatibility. The dynamic nature of kidney exchanges were explored by Ünver (2010), Akbarpour, Li, and Gharan (2013), and Anderson et al. (2017).

Besides its contributions to the practice and theory of kidney exchange, our paper contributes to the emerging field of market design by bringing to light a potential unintended consequence of the 2014 US deceased-donor allocation reform in the form of a reduction in the number of living-donor transplants, and by making easy-to-implement welfare-enhancing policy recommendations to avoid this potential loss. ${ }^{8}$

The organization of the rest of the paper is as follows: In Section 2 we further explain the

\footnotetext{
${ }^{8}$ There are also other papers in the market design literature that have shown unintended consequences of well-intentioned new policies. E.g., see Dur et al. (2016) in school choice context and Sönmez (2013) in army branch and officer matching for ROTC in the US.
} 
preliminaries, In Section 3 we introduce the model, and in Section 4 we analyze the impact of availability and timing of various blood subtyping technologies on the number of living-donor kidney transplants. In Section 5, we show via computational simulations that all our theoretical results are verified using kidney-donation data from the US. In Section 6, we conclude. The Appendix is devoted to proofs (Appendix A), the extended analysis allowing for 3-way kidney exchange in addition to 2-way exchange (Appendix B), additional results related to simulations (Appendix C).

\section{Formation of the Kidney-Exchange Pool}

A kidney patient arrives to a hospital with his living donor. Using the testing technology available to it, the hospital conducts a number of tests to determine whether the pair is compatible or not. If the pair is deemed compatible (with the available testing technology) then the donor directly donates to the patient. Otherwise the pair is transferred to a kidney exchange pool for a potential exchange of donors between patients with incompatible donors.

For a patient to be deemed medically compatible with his donor, the pair should be both

1. tissue-type compatible, and

2. blood-type compatible.

\subsection{Tissue-Type Compatibility}

Of the two potential medical barriers to transplantation, tissue-type incompatibility (a.k.a. "positive crossmatch") is the less structured one, and it has to do with the patient having preformed antibodies against one or more of the donor's human leukocyte antigen (HLA) proteins. Antibodies can arise from exposure to foreign proteins, e.g., through prior transplants, blood transfusions, or even childbirth. (Consequently, mothers are less likely to be compatible with a kidney from the father of their children than from a random donor from the same population.) The positive crossmatch probability between a random patient-donor pair is relatively low, with a mean probability of 11 percent as reported by Zenios, Woodle, and Ross (2001), although this probability is not uniform across all patients. Each individual's tissue type incompatibility rate is summarized through a probability against a random donor, known as panel reactive antibody (PRA) score. This number tells the percentage of random donors from a benchmark population a patient would be tissue-type incompatible with. (The mean of these scores in the population is around 11 percent.) In the medical literature, we could not find a more detailed PRA distribution for the population different from this mean statistic (although empirically 
it is possible to calculate the PRA distribution using different data sets for various types of individuals; for example, waitlisted kidney patients using UNOS data).

\subsection{Blood-Type Compatibility}

Blood-type compatibility is the more structured of the two primary medical requirements for kidney transplantation. The standard blood type classification is known as ABO grouping. Under this classification there are two types of red blood cell proteins (or antigens) referred to as antigen A and antigen B. An individual can have any combination of these two antigens, and individuals produce antibodies against the antigens they lack. There are four blood types determined by the presence or absence of these two antigens on the surface of red blood cells:

1. Blood type O: Has neither A nor B antigens on red cells (but both antibody anti-A and antibody anti-B are in the plasma).

2. Blood type A: Has only the A antigen on red cells (and antibody anti-B in the plasma).

3. Blood type B: Has only the B antigen on red cells (and antibody anti-A in the plasma).

4. Blood type AB: Has both A and B antigens on red cells (but neither antibody anti-A nor antibody anti-B in the plasma).

The above-described blood type classification induces the standard ABO blood-type compatibility, or simply ABO compatibility where:

- blood type O patients can receive a kidney only from blood type O donors,

- blood type A patients can receive a kidney from blood type $\mathrm{A}$ and $\mathrm{O}$ donors,

- blood type B patients can receive a kidney from blood type B and O donors, and

- blood type $\mathrm{AB}$ patients can receive a kidney from all donors.

We will treat ABO compatibility as the baseline technology that determines the kidney-exchange pool along with tissue-type compatibility.

The immunologic asymmetry under ABO compatibility has historically resulted in an unfavorable situation for blood type $\mathrm{O}$ patients in the form of less access to transplant kidneys compared to blood type $\mathrm{A}$ or $\mathrm{AB}$ patients. In addition to blood type $\mathrm{O}$ patients, blood type $\mathrm{B}$ patients are also biologically disadvantaged because they are more likely to suffer from kidney disease. In part due to this uneven access to transplant kidneys under the ABO-compatibility technology, a more refined classification of blood types has started to play an important role in the allocation of deceased-donor kidneys in the US since 2014. 


\subsection{Blood Subtype A2/A2B Compatibility}

Unlike the red blood cell antigen $\mathrm{B}$, antigen $\mathrm{A}$ is not a single antigen and it consists of two major subtypes, A1 and A2. When transplanted to a blood type B or O patient, a subtype A2 kidney generates considerably weaker antibody response than a subtype A1 kidney. Similarly, subtype A2B kidneys (those with both subtype A2 and type B antigens) generate weaker antibody response than A1B kidneys (those with both subtype A1 and type B antigens) in blood type B patients. The antibody response to subtype A2 (or A2B) kidneys is not uniform across all blood type $\mathrm{B}$ or $\mathrm{O}$ patients, and it depends on patients' antibody anti-A (IgG) titer values. Transplantation of subtype A2 kidneys to patients with titer values less than 1:8 is considered safe in the US. With the titer distribution of the US patient population, that means

- subtype A2 (or A2B) kidneys can be safely transplanted to 80 percent of blood type B patients, and

- subtype A2 kidneys can be safely transplanted to 30-40 percent of blood type O patients.

Blood type A subtyping technology provides a unique opportunity to increase transplant access for biologically disadvantaged candidates from blood types B and O. And, indeed, since 2014, subtype A2 and A2B deceased-donor kidneys have been preferentially allocated in the US to blood type B patients for this very reason. However, transplantation of a subtype A2 kidney to a type $\mathrm{B} / \mathrm{O}$ patient (or transplantation of a subtype $\mathrm{A} 2 \mathrm{~B}$ kidney to a type $\mathrm{B}$ patient) requires a number of additional tests for both the patient and the donor:

1. Antibody Anti-A Titer Value Tests for Patients: Patient antibody anti-A (IgG) titer value should be consistently below a certain threshold over a period of time, often over the last 6 months.

2. Subtyping Tests for Blood-Type A Donors: Due to the unreliability of a single test, the following two tests are conducted to determine the subtype of a blood type A donor.

(a) Preliminary subtyping test: This test is not completely reliable by itself, and there is a 3.5 percent chance that an A1 kidney will test as A2 (Bryan et al., 2006).

(b) Confirmatory subtyping test: The second test reduces the probability of mistakenly identifying an A1 kidney as A2 to 0.032 percent.

With the recent changes in the deceased-donor kidney-allocation rule in the US, blood type B patients are regularly tested for their antibody anti-A titer values in order to benefit from the preferential allocation of A2/A2B kidneys. Hence, a blood type B patient's antibody anti-A titer value history becomes readily available when he is transferred to a kidney-exchange pool 
with an incompatible donor. That in turn means that he not only has access to subtype A2/A2B kidneys from the deceased-donor list but also from the kidney-exchange pool. In contrast, a blood type $\mathrm{O}$ patient often lacks the required antibody anti-A titer value history upon being transferred to a kidney-exchange pool with an incompatible donor. And lacking the titer value history, he is not qualified to receive a subtype A2 kidney via exchange for at least 6 months. Because subtype A2 kidneys can be transplanted to blood type B or O patients only in the presence of an established (and low) titer value history, we will treat blood type A subtyping technology as a combination of the following two separate technologies:

1. A2-to-B compatibility: This technology extends the standard ABO-compatibility technology by allowing subtype A2/A2B kidneys to be transplanted to qualified blood type B patients.

A2-to-B compatibility technology is the best approximation of the current practice in the US where antibody anti-A titer value history is systematically established only for blood type B patients.

2. A2-to-O compatibility: This technology extends the standard ABO-compatibility technology by allowing subtype A2 kidneys to be transplanted to qualified blood type O patients.

A2-to-O compatibility technology corresponds to a hypothetical scenario where antibody anti-A titer value history is systematically established only for blood type O patients.

We will also consider the following combined subtyping technology:

3. Full compatibility: This technology extends ABO compatibility by allowing subtype A2 kidneys to be transplanted to all qualified blood type B/O patients, and subtype A2B kidneys to all qualified blood type B patients.

This final technology corresponds to the hypothetical scenario where an antibody antiA titer history is systematically established for both blood type B and blood type O patients.

\section{$3 \quad$ The Model}

A pair of type X-Y denotes a patient-donor pair with an X-blood-type patient and a Y-bloodtype (or subtype) donor. For our baseline scenario, the hospital conducts the tissue-type compatibility test as well as the baseline ABO-compatibility test upon the arrival of a patient-donor pair to determine whether they are compatible or not. No subtyping technology is available 
under the baseline scenario. Patients who are both tissue-type and blood-type compatible with their donors receive a kidney transplant directly from their compatible donors. In this baseline scenario, patients who are either tissue-type incompatible or blood-type incompatible are transferred to a single kidney-exchange pool. This process determines the composition of the kidney-exchange pool.

In addition to the baseline ABO-compatibility technology, we also consider the three subtyping technologies A2-to-B compatibility, A2-to-O compatibility, and full compatibility, described in Section 2.3. When available, a subtyping technology will replace the baseline ABOcompatibility technology, thereby potentially removing a barrier to direct donation. Therefore compared to the baseline ABO compatibility, the availability of each of the three subtyping technologies will potentially extend the set of patients who receive a transplant via direct donation and potentially shrink the set of patients who are transferred to the kidney-exchange pool.

A 2-way kidney exchange involves two patients, each of whom is compatible with the other patient's donor. When a 2-way exchange is carried out, both patients receive a kidney from the other patient's donor. Once the kidney-exchange pool forms under any of the abovedescribed bloodtyping/subtyping technologies, a maximal-size kidney exchange is determined for the given pool of incompatible patient donor pairs as in Roth, Sönmez, and Ünver (2005b, 2007). Our focus is to analyze the impact of availability of various subtyping technologies on the total number of living-donor transplants, including direct transplants from patients' compatible donors and transplants from kidney exchanges.

Following Roth, Sönmez, and Ünver (2007), we rely on the following three assumptions about the composition of the kidney-exchange pool to derive our analytical results. While these assumptions are used for our analytical results, they will be dispensed with in our simulations in Section 5. We will observe that all qualitative implications of our analytical results are verified by our simulations in the absence of these assumptions. We next motivate and formally state these assumptions.

Let $\mathrm{X}, \mathrm{Y}$ be two distinct blood types such that a blood type $\mathrm{Y}$ donor can donate to a blood type $\mathrm{X}$ patient. Being blood-type compatible, a pair of type $\mathrm{X}-\mathrm{Y}$ is only transferred to the kidney-exchange pool when the pair is tissue-type incompatible, a relatively rare event. In contrast, a pair of the opposite type $\mathrm{Y}-\mathrm{X}$ is always transferred to the kidney-exchange pool because they are blood-type incompatible. Therefore, in the long run, there will be an abundance of $\mathrm{Y}-\mathrm{X}$ pairs in the kidney-exchange pool in comparison with $\mathrm{X}-\mathrm{Y}$ pairs, and thus it will not be possible to match all pairs of type $\mathrm{Y}-\mathrm{X} .{ }^{9}$ We refer to this situation as a pair of

\footnotetext{
${ }^{9}$ That is why there is an abundance of blood type $\mathrm{O}$ patients with non-O donors in kidney-exchange pools all over the world.
} 
type Y-X being on the long side of the exchange. This simple observation, which is also empirically observed throughout the world in kidney-exchange programs, is the basis of our first assumption.

\section{Assumption 1 (Large Population Assumption (LP)):}

(i) Under $A B O$ compatibility, pairs of types $O-A, O-B, O-A B, A-A B$ and $B-A B$ are on the long side of the exchange.

(ii) Under A2-to-B compatibility, pairs of types $O-A, O-B, O-A B, A-A B$ and $B-A 1 B$ are on the long side of the exchange.

(iii) Under $A 2$-to- $O$ compatibility, pairs of types $O-A 1, O-B, O-A B, A-A B$ and $B$ - $A B$ are on the long side of the exchange.

(iv) Under full compatibility, pairs of types $O-A 1, O-B, O-A B, A-A B$ and $B-A 1 B$ are on the long side of the exchange.

Our next assumption is based on the following empirical observation for the US: The frequency of types A-B and B-A are 0.05 and 0.03 respectively (Terasaki, Gjertson, and Cecka, 1998).

Assumption 2 (Type Frequencies Assumption (TF)): There are at least as many type $A-B$ pairs as type $B-A$ pairs.

While patients can have tissue-type incompatibility with their own donors, to establish an upper bound on the number of possible transplants we will assume that they are tissue-type compatible with other donors. In the same spirit, we will assume that all blood type B patients are qualified to receive subtype A2/A2B kidneys, and all blood type O patients are qualified to receive subtype A2 kidneys.

\section{Assumption 3 (Upper-Bound Assumption (UB)):}

(i) No patient is tissue-type incompatible with another patient's donor.

(ii) Each patient in the exchange pool has an IgG antibody titer value less than 1:8.

We previously mentioned that there is around 89 percent chance that a random patient and donor are tissue-type compatible. In the Introduction, we also mentioned that about 80 percent of blood-type B patients and 30-40 percent of blood-type O patients have the required low IgG antibody titer values for A2/A2B and A2 donor subtype compatibility, respectively. This assumption takes these rates to be 100 percent (except the tissue incompatibility probability of a patient with his own donor).

When we later dispense with this assumption in our simulations, we will see that, in large pools, this assumption is not very consequential. The reason is that, although a patient who 
is tissue-type incompatible with his own donor will likely have positive crossmatches with some other patients' donors also, in a sufficiently large pool there will also be many donors with whom he has no tissue-type incompatibility. ${ }^{10}$ Similarly, while many blood type B or O patients will have high antibody anti-A ( $\operatorname{IgG})$ titer values that disqualify them from receiving subtype A2/A2B kidneys, there will also be many patients on the long side of the market who can receive those kidneys instead.

\section{Analytical Results under 2-way Exchange}

For a given set $T$ of patient-donor pairs, let \#T denote the cardinality of set $T$. Let $(X-Y)_{\checkmark}$ denote the set of tissue-type-compatible pairs of type $\mathrm{X}-\mathrm{Y}$, and $(X-Y)_{\mathrm{x}}$ denote the set of tissue-type-incompatible pairs of type X-Y (i.e. those with positive crossmatch) respectively. For any nonnegative number $k$, let $\lfloor k\rfloor$ denote the integer part of $k$, i.e., the greatest integer no larger than $k$. Given a set $T$, let $\mathbf{o d d} \mathbf{d}_{T}$ be equal to 1 if the cardinality of $T$ is odd, and 0 if it is even.

\subsection{Timing of Subtyping Tests: Before Joining Kidney-Exchange Pool}

Under the current regulations of the OPTN, all tests pertaining to patient-donor compatibility, including the subtyping tests for blood type A donors, are conducted at the hospital once a patient-donor pair arrives (Section 14.5 of Organ Procurement and Transplantation Network, 2016). Assuming 2-way exchange only, in our first three results we analyze how the number of living-donor transplants is affected under the current timing of medical tests when the baseline ABO-compatibility technology is replaced with each of the three subtyping technologies we formulated in Section 2.3.

We first consider the impact of replacing the ABO-compatibility technology with the A2to-B subtyping technology. As we argued in Section 2.3, A2-to-B technology is a good approximation of the present implementation of the blood type A subtyping technology, and thus our first comparative statics exercise captures the de facto impact of the 2014 policy reform on the number of living-donor kidney transplants. One might be tempted to presume that removing a barrier to transplantation through utilization of a new and improved technology will necessarily increase the number of living-donor transplants. As our first comparative-statics exercise shows, this is not necessarily the case.

\footnotetext{
${ }^{10}$ As in our simulations, in a model where tissue-type incompatibility is determined through a fixed probability between each patient and donor, this is a consequence of the well-known Erdös and Rényi (1960) theorem when the size of the exchange pool is sufficiently large.
} 
Proposition 1 Assume LP(i), LP(ii), TF, UB. Consider the 2-way-exchange policy. If the compatibility technology changes from ABO compatibility to A2-to- $B$ compatibility, then

(i) the number of transplants via direct donation increases by $\#(B-A 2)_{\checkmark}+\#(B-A 2 B)_{\checkmark}$,

(ii) the number of transplants via exchange changes by

$$
-2 \#(B-A 2)_{\checkmark}+\#(A B-A 2 B)_{x}+\#(B-A 2 B)_{x}+\Lambda, \text { and }
$$

(iii) the total number of transplants changes by

$$
-\#(B-A 2)_{\checkmark}+\#(A B-A 2 B)_{x}+\#(B-A 2 B)+\Lambda
$$

where $\Lambda=\left(\operatorname{odd}_{(A B-A B)_{x}}-\operatorname{odd}_{(A B-A 1 B)_{x}}\right)+\left(\operatorname{odd}_{(B-B)_{x}}-\operatorname{odd}_{(B-A 2 B)_{x} \cup(B-B)_{x}}\right)$.

Observe that $\Lambda$ is negligible in a large pool, and the very low frequency of subtype A2B individuals in the population implies that the de facto practiced A2-to-B subtyping technology is detrimental to living donor kidney transplantation. The A2B subtype exists only in 0.8 percent of the US population, making the changes denoted in parts (ii) and (iii) of the proposition negative under any plausible scenario of pair formation for any realistic pool size. While the number of direct transplants increases upon adoption of the A2-to-B technology, the number of transplants from kidney exchange decreases even more, thus reducing the total number of living-donor transplants. The intuition for this result is quite simple: While all pairs of type B-A are transferred to the kidney-exchange pool in the absence of a subtyping technology, they are all subsequently matched at the kidney-exchange pool with pairs of the opposite type A-B, which is on the long side of the exchange by Assumption TF. Thus, when a subset of type B-A patients receive direct transplants from their own donors utilizing the A2-to-B technology, there is no net benefit to members of type B-A, but there is a net harm to members of the opposite type A-B. On the other hand, the number of transplants via direct donation increases by the compatible B-A2B pairs without any spillover on the exchange. This is due the fact that $\mathrm{B}-\mathrm{AB}$ pairs are on the long side under ABO compatibility by Assumption LP(i), and these direct donations only curb the long side of the market. Moreover, A2-to-B subtyping technology allows for exchanges among incompatible B-A2B pairs and between AB-A2B and B-A1B pairs (which are not possible under ABO compatibility). The crucial observation is that these additional benefits in the live donation context (via compatible B-A2 and B-A2B pairs) and the exchange context (via incompatible AB-A2B and B-A2B pairs) are insufficient to compensate the efficiency loss due to unmatched A-B pairs.

In our second comparative-statics exercise, we consider the impact of replacing the ABOcompatibility technology with the A2-to-O subtyping technology. As we argue in Section 2.3, A2-to-O subtyping technology corresponds to a hypothetical scenario where antibody anti-A 
titer value testing becomes routine for blood type $\mathrm{O}$ patients but not for blood type B patients. The impact of this hypothetical scenario is in striking contrast with the detrimental impact of the current de facto implementation of A2-to-B subtyping technology.

Proposition 2 Assume LP(i), LP(iii), UB. Consider the 2-way-exchange policy. If the compatibility technology changes from ABO compatibility to A2-to-O compatibility, then

(i) the number of transplants via direct donation increases by $\#(O-A 2)_{\checkmark}$,

(ii) the number of transplants via exchange increases by $\#(O-A 2)_{x}+\#(A-A 2)_{x}+\Theta$, and (iii) the total number of transplants increases by $\#(O-A 2)+\#(A-A 2)_{x}+\Theta$, where $\Theta=\left(\boldsymbol{o d d}_{(O-O)_{x}}-\boldsymbol{o d d}_{(O-O)_{x} \cup(O-A 2)_{x}}\right)+\left(\boldsymbol{o d d}_{(A-A)_{x}}-\boldsymbol{o d d}_{(A-A 1)_{x}}\right)$

Unlike the detrimental impact of the A2-to-B subtyping technology on the composition of the kidney-exchange pool, the A2-to-O subtyping technology improves it. That happens through new types of both direct donation and also exchange, which are not possible under ABO compatibility: First, the number of O-A pairs (that are on the long side of the exchange under ABO compatibility) is curbed via direct donations by the compatible O-A2 pairs. Second, an incompatible O-A2 pair is not on the long side of the exchange by Assumption LP(iii) and can be matched with an $\mathrm{O}-\mathrm{O}$ pair, and this increases the number of transplants via exchange by the number of incompatible O-A2 pairs. Third, each incompatible A-A2 pair can be matched with an O-A1 pair, which is on the long side by Assumption LP(iii). Since A-A pairs are matched with each other under ABO compatibility, this increases the number of transplants via exchange by the number of incompatible A-A2 pairs.

Our first two results have an important policy implication on the welfare effects of integrating blood type A subtyping technology to an exchange: Under the current protocol of subtype testing by hospitals, A2-to-B compatibility technology leads to a reduction in the number of transplants, whereas the counterfactual A2-to-O compatibility technology leads to an increase. This observation points to a crucial difference between deceased donation and kidney exchange. While the preferential allocation of subtype A2 deceased-donor kidneys to blood type B patients is only a distributional matter, directly or indirectly extending the same policy to kidney exchange has a detrimental effect on the overall welfare of the patient population. In our simulations we show that this detrimental effect on patient welfare is not restricted to specific ethnic groups but rather shared across all ethnicities, including those who are meant to benefit from the preferential allocation of subtype A2 kidneys to blood type B patients.

In the context of 2-way exchange, the effect of A2-to-B compatibility on direct donation and that of A2-to-O compatibility are mutually exclusive. Similarly, the sets of pairs affected in exchange by A2-to-B compatibility and by A2-to-O compatibility are mutually exclusive. Thus, the effect of replacing ABO compatibility with full compatibility on the number of living-donor 
transplants is simply the direct sum of the individual effects of these two subtyping technologies, resulting in the following easy result.

Proposition 3 Assume LP(i), LP(iv), TF, UB. Consider the 2-way-exchange policy. If the compatibility technology changes from ABO compatibility to full compatibility, then

(i) the number of transplants via direct donation increases by

$$
\#(O-A 2)_{\checkmark}+\#(B-A 2)_{\checkmark}+\#(B-A 2 B)_{\checkmark}
$$

(ii) the number of transplants via exchange changes by

$\#(O-A 2)_{x}+\#(A-A 2)_{x}-2 \#(B-A 2)_{\checkmark}+\#(A B-A 2 B)_{x}+\#(B-A 2 B)_{x}+\Theta+\Lambda$, and

(iii) the total number of transplants changes by

$$
\#(O-A 2)+\#(A-A 2)_{x}-\#(B-A 2)_{\checkmark}+\#(A B-A 2 B)_{x}+\#(B-A 2 B)+\Theta+\Lambda,
$$

where $\Theta=\left(\operatorname{odd}_{(O-O)_{x}}-\boldsymbol{o d d}_{(O-O)_{x} \cup(O-A 2)_{x}}\right)+\left(\operatorname{odd}_{(A-A)_{x}}-\boldsymbol{o d d}_{(A-A 1)_{x}}\right)$ and $\Lambda=\left(\boldsymbol{o d d}_{(A B-A B)_{x}}-\right.$ $\left.\operatorname{odd}_{(A B-A 1 B)_{x}}\right)+\left(\operatorname{odd}_{(B-B)_{x}}-\operatorname{odd}_{(B-A 2 B)_{x} \cup(B-B)_{x}}\right)$.

The term $\Theta+\Lambda$ is negligible in a large pool, and blood type B has lower representation in the population than both blood type A and blood type O. Thus, the net welfare effect of replacing ABO-compatibility technology with full-compatibility technology is positive. Compared to adopting the hypothetical A2-to-O technology, adopting full-compatibility technology is easier because it simply requires establishing antibody anti-A titer value history for blood type $\mathrm{O}$ patients in addition to the current practice of establishing it for blood type $\mathrm{B}$ patients only. While adoption of the A2-to-O technology results in higher welfare for the patient population, it is harder to implement because it either requires explicit preferential allocation of A2 kidneys to blood type $\mathrm{O}$ patients in kidney exchange or implicit preferential allocation of $\mathrm{A} 2$ kidneys to blood type $\mathrm{O}$ patients in kidney exchange by collecting antibody anti-A titer value history for blood type $\mathrm{O}$ patients only (in direct conflict with the 2014 reform).

We can summarize the results of this section as follows: For the present timing of the subtyping tests, while it is easy to improve upon the current de facto practice of A2-to-B technology, it is harder to implement the highest-welfare subtyping technology, A2-to-O. To put it differently, obtaining the most favorable kidney-exchange pool composition might be a potentially challenging policy task under the present timing of subtyping tests. This observation motivates the following consideration: What if blood type A subtyping tests were delayed until after blood- or tissue-type-incompatible pairs are transferred to the kidney-exchange pool? Could this tweak further improve the welfare of the patient population? We answer this question in the affirmative in the next section. 


\subsection{Timing of Subtyping Tests: After Joining Kidney-Exchange Pool}

As we indicated before, transplanting a subtype A2 kidney to a blood type B or O patient (or a subtype A2B kidney to a blood type B patient) requires conducting additional tests both for the patient and also for his donor. If a blood type $\mathrm{B}$ or $\mathrm{O}$ patient desires to be considered for a subtype A2 kidney, it is the responsibility of his hospital to generate an antibody anti-A titer value history for him. Similarly, it is the responsibility of his hospital to determine the subtype of his blood type A donor if the pair desires to utilize the donor as a subtype A2 or A2B donor (either by direct transplantation or via an exchange).

In this section, we focus on the impact of deferring this second set of subtyping tests until after a blood- or tissue-type-incompatible pair is transferred to the kidney-exchange pool. As such, unlike in Section 4.1, the composition of the kidney-exchange pool remains identical across all four blood typing/subtyping technologies considered. Observe that, this would also mean there would be some compatible pairs in the kidney-exchange pool although this subtypingenabled compatibility would only be determined once a pair arrived to the kidney-exchange pool. Hence, in this section, we will maximize the number of transplants in the kidney-exchange pool not only through 2-way kidney exchanges, but also by allowing direct transplants to patients from their subtype A2 or A2B donors. ${ }^{11}$

Assuming 2-way exchange only, in our next three results we analyze how the maximum number of living-donor transplants changes under this deferred timing of blood type A subtyping tests when the baseline ABO-compatibility technology is replaced with each of the three subtyping technologies we formulated in Section 2.3.

We first consider the impact of replacing the ABO-compatibility technology with the A2to-B subtyping technology under the delayed timing of blood type A subtyping tests.

Proposition 4 Assume LP(i), LP(ii), TF, UB. Consider the 2-way-exchange policy when the subtyping test for the donor is conducted after a pair joins the exchange pool. If the compatibility technology changes from ABO compatibility to A2-to-B compatibility, then

(i) the number of transplants via direct donation increases by

$$
\max \left\{0, \#(B-A 2 B)_{\checkmark}-\boldsymbol{o d d}_{(B-A 2 B)_{x} \cup(B-B)_{x}}\right\}
$$

(ii) the number of transplants via exchange increases by

$$
\#(A B-A 2 B)_{x}+\#(B-A 2 B)_{x}+\min \left\{\#(B-A 2 B)_{\checkmark}, \boldsymbol{o d d}_{(B-A 2 B)_{x} \cup(B-B)_{x}}\right\}+\Lambda^{\prime}, \text { and }
$$

\footnotetext{
${ }^{11}$ The problem of maximizing the total number of transplants via direct transplants and 2-way exchanges in a kidney-exchange pool that includes compatible pairs is studied in Sönmez and Ünver (2014).
} 
(iii) the total number of transplants increases by

$$
\#(B-A 2 B)+\#(A B-A 2 B)_{x}+\Lambda^{\prime}
$$

where

$$
\begin{aligned}
\Lambda^{\prime}= & \left(\operatorname{odd}_{(A B-A B)_{x}}-\boldsymbol{o d d}_{(A B-A 1 B)_{x}}\right)+ \\
& \left(\boldsymbol{o d d}_{(B-B)_{x}}-\left(1-\min \left\{1, \#(B-A 2 B)_{\checkmark}\right\}\right) \operatorname{odd}_{(B-A 2 B)_{x} \cup(B-B)_{\times}}\right) .
\end{aligned}
$$

The key observation for Proposition 4 is that the de facto practiced A2-to-B subtyping technology is no longer detrimental to living-donor kidney transplantation once utilization of the subtyping technology is deferred until after the formation of the kidney-exchange pool. This is, of course, expected because the removal of a barrier to direct transplantation can only decrease the number of living-donor transplants through an unfavorable effect on the composition of the kidney-exchange pool. The proof basically follows from characterizing all exchanges, which are not possible under ABO compatibility. The B-A2B pairs are actually like B-B pairs and they are not on the long side of the market under A2-to-B compatibility by Assumption LP(ii). Thus, each B-B or B-A2B pair can be matched with any pair from the same group by Assumption UB. If the number of incompatible B-A2B and B-B pairs are even, then all compatible B-A2B pairs can be set for direct donation; otherwise, all but one are set for direct donation and only one is set for an exchange with an incompatible B-B or B-A2B pair. Also, while an incompatible B-A2B pair is on the long side under ABO compatibility by Assumption LP(i), it is not on the long side under A2-to-B compatibility by Assumption LP(ii). Thus, under A2-to-B compatibility, since each incompatible B-A2B pair can be matched with a B-B pair, the number of transplants via exchange increases by the number of incompatible B-A2B pairs. Similarly, each incompatible AB-A2B pair is like an AB-B pair and can be matched with a B-A1B pair, which is on the long side by Assumption LP(ii), and is matched with another AB-AB pair under ABO compatibility. Thus, since all AB-AB pairs are matched with each other under $\mathrm{ABO}$ compatibility, the number of transplants via exchange increases by the number of incompatible AB-A2B pairs under A2-to-B compatibility.

We next consider the impact of replacing the ABO-compatibility technology with the A2to-O subtyping technology under the delayed timing of blood type A subtyping tests.

Proposition 5 Assume LP(i), LP(iii), UB. Consider the 2-way-exchange policy when the subtyping test for the donor is conducted after a pair joins the exchange pool. If the compatibility technology changes from ABO compatibility to A2-to-O compatibility, then

(i) the number of transplants via direct donation increases by

$$
\max \left\{0, \#(O-A 2)_{\checkmark}-\boldsymbol{o d d}_{(O-O)_{x} \cup(O-A 2)_{x}}\right\}
$$


(ii) the number of transplants via exchange increases by

$$
\#(A-A 2)_{x}+\#(O-A 2)_{x}+\min \left\{\#(O-A 2)_{\checkmark}, \boldsymbol{o d d}_{(O-O)_{x} \cup(O-A 2)_{x}}\right\}+\Theta^{\prime}, \text { and }
$$

(iii) the total number of transplants increases by $\#(O-A 2)+\#(A-A 2)_{x}+\Theta^{\prime}$, where

$$
\begin{aligned}
\Theta^{\prime}= & \left(\operatorname{odd}_{(A-A)_{\times}}-\boldsymbol{o d d}_{(A-A 1)_{\times}}\right)+ \\
& \left(\boldsymbol{o d d}_{(O-O)_{x}}-\left(1-\min \left\{1, \#(O-A 2)_{\checkmark}\right\}\right) \operatorname{odd}_{(O-O)_{x} \cup(O-A 2)_{\times}}\right) .
\end{aligned}
$$

Recall that unlike the A2-to-B subtyping technology, utilization of the A2-to-O subtyping technology does not affect the composition of the kidney-exchange pool in an unfavorable way. As such, deferral of the subtyping tests only marginally affects the number of living-donor transplants through the residual term $\Theta^{\prime}$ under this technology. The (marginal) difference is due the fact that if the total number of incompatible O-A2 and O-O pairs is odd, then only one such pair remains unmatched with a pair from the pool of incompatible O-A2 pairs and O-O pairs, and in this case, a subtype-compatible O-A2 pair, if any, should be matched with that remaining pair to prevent any such loss.

We finally consider the impact of replacing the ABO-compatibility technology with fullcompatibility technology under the delayed timing of blood type A subtyping tests. As in the case of Proposition 3, the effect of replacing ABO compatibility with full compatibility on the number of living-donor transplants is simply the sum of the individual effects of A2-to-B and A2-to-O technologies.

Proposition 6 Assume $L P(i), L P(i v), T F, U B$. Consider the 2-way-exchange policy when the subtyping test for the donor is conducted after a pair joins the exchange pool. If the compatibility technology changes from ABO compatibility to full compatibility, then

(i) the number of transplants via direct donation increases by

$$
\begin{aligned}
& \max \left\{0, \#(B-A 2 B)_{\checkmark}-\boldsymbol{o d d}_{(B-A 2 B)_{x} \cup(B-B)_{x}}\right\}+ \\
& \max \left\{0, \#(O-A 2)_{\checkmark}-\boldsymbol{o d d}_{(O-O)_{x} \cup(O-A 2)_{x}}\right\}
\end{aligned}
$$

(ii) the number of transplants via exchange increases by

$$
\begin{aligned}
& \#(A B-A 2 B)_{x}+\#(B-A 2 B)_{x}+\min \left\{\#(B-A 2 B)_{\checkmark}, \boldsymbol{o d d}_{(B-A 2 B)_{x} \cup(B-B)_{x}}\right\}+\Lambda^{\prime}+ \\
& \#(O-A 2)_{x}+\#(A-A 2)_{x}+\min \left\{\#(O-A 2)_{\checkmark}, \boldsymbol{o d d}_{(O-O)_{x} \cup(O-A 2)_{x}}\right\}+\Theta^{\prime}, \text { and }
\end{aligned}
$$

(iii) the total number of transplants increases by

$$
\#(O-A 2)+\#(A-A 2)_{x}+\#(A B-A 2 B)_{x}+\#(B-A 2 B)+\Theta^{\prime}+\Lambda^{\prime}
$$


where

$$
\begin{aligned}
\Lambda^{\prime}= & \left(\boldsymbol{o d d}_{(A B-A B)_{\times}}-\boldsymbol{o d d}_{(A B-A 1 B)_{\times}}\right)+ \\
& \left(\boldsymbol{o d d}_{(B-B)_{x}}-\left(1-\min \left\{1, \#(B-A 2 B)_{\checkmark}\right\}\right) \operatorname{odd}_{(B-A 2 B)_{x} \cup(B-B)_{\times}}\right),
\end{aligned}
$$

and

$$
\begin{aligned}
\Theta^{\prime}= & \left(\operatorname{odd}_{(A-A)_{x}}-\boldsymbol{o d d}_{(A-A 1)_{x}}\right)+ \\
& \left(\boldsymbol{o d d}_{(O-O)_{x}}-\left(1-\min \left\{1, \#(O-A 2)_{\checkmark}\right\}\right) \boldsymbol{o d d}_{(O-O)_{x} \cup(O-A 2)_{\times}}\right) .
\end{aligned}
$$

The key takeaway in this section is that, with the deferred timing of the blood type A subtyping tests, the full-compatibility technology becomes the highest welfare implementation of the advancement in blood type testing technology. And implementation of this best utilization of subtyping technology is realistic because it simply requires generating a history of antibody anti-A titer value history for blood type $\mathrm{O}$ patients, as in the case of blood type B patients, and deferring subtyping tests of blood type A donors until after they join the kidney-exchange pool.

\section{$4.3 \quad 2 \& 3$-way Exchanges}

When 3-way exchanges are feasible as well, they potentially compensate the efficiency loss (in Proposition 1) due to the compatible B-A2 pairs not participating in the exchange. This potential recovery of efficiency depends on the distribution of the pairs in the pool: A 3-way exchange with a B-O pair, an O-A pair, and A-B pair (or with an AB-A pair, an A-B pair, and a B-AB pair) possibly matches an A-B pair that remains unmatched with a B-A pair via a 2-way exchange. A very rough intuition is that, if the number of $\mathrm{B}-\mathrm{O}$ and $\mathrm{AB}-\mathrm{A}$ pairs is too small, under ABO compatibility, each of these pairs is matched with an A-B pair via a 3-way exchange, and an unmatched A-B pair (due to B-A2 pairs not participating the exchange) still remains unmatched under A2-to-B compatibility. In this case, efficiency is not recovered at all. On the other hand, if the pool of B-O and AB-A pairs is sufficiently large, the efficiency loss is fully compensated. We provide a detailed analysis of 3-way exchanges by comparing ABO compatibility with A2-to-B compatibility in Appendix B. For A2-to-O compatibility, the results for 2-way exchanges are maintained for 3-way exchanges: The subtype technology does not cause any efficiency loss (Appendix B).

\section{Simulations}

In this section, we report the results of simulations using the US patient statistics obtained from OPTN database to demonstrate the magnitudes of potential welfare benefits and harms 
of subtype A2 matching with $\mathrm{B}$ or $\mathrm{O}$ patients in an exchange under a number of different scenarios.

\subsection{Simulation Setup}

In each simulation, we randomly generate $n$ non-blood related patient-donor pairs as follows: Each patient is represented by a set of characteristics, namely, his ethnic background (i.e., his race), his blood type, his A2 subtype compatibility status (for $\mathrm{O}$ and B blood type patients), and his probability of having tissue type incompatibility with a random donor (known as his panel reactive antibody or simply PRA level). Each patient is assumed to arrive paired with a non-biologically-related donor. The donor can be either his spouse or another donor. If she is his spouse, then we assume she has the same race as the patient. Otherwise, we randomly generate her race using the US adult population race statistics. Based on her race, we randomly and independently determine her other characteristics, such as her blood type and A2 status (if she is A blood type). The probability distributions used are reported in Table 1.

In each simulation, after a patient-donor pair is generated, if the donor is compatible with the patient (under regular ABO compatibility), she immediately donates to her patient and the pair does not participate in the exchange. Otherwise the pair becomes a potential entrant to the exchange. ${ }^{12}$

In this section we drop the large-market assumption that we used in our theoretical analysis of Section 4. Hence, each patient can have tissue-type incompatibility with not only his own donor but also other donors. Depending on the PRA of the patient as low, medium, or high, which shows how likely a patient is to develop tissue-type incompatibility with a randomly selected donor from the US population, his donor and any other blood-type-compatible donor are independently tested for tissue-type incompatibility. A PRA value below 10 percent is considered low; PRA values between 10-80 percent are considered medium; and PRA values above 80 percent are considered high, according to the OPTN annual report. ${ }^{13}$

Frequencies of low, medium, and high PRA patients reported in the OPTN database are given in Table 1. A more detailed PRA distribution is unavailable in the medical literature. Thus, we assume that:

- each low-PRA patient has a tissue-type incompatibility probability of 5 percent with a random donor,

\footnotetext{
${ }^{12}$ We use the same blood type distribution for both the donors and patients of a given race.

${ }^{13}$ These statistics refer to the PRA values of the existing patients on the deceased-donor waiting list. As mentioned in Section 2.1, the population PRA distribution is not readily available.
} 


\begin{tabular}{|c|r|r|r|r|r|}
\hline & White & Black & Asian & $\begin{array}{r}\text { Amer. } \\
\text { Indian }\end{array}$ & $\begin{array}{r}\text { Pacific } \\
\text { Island. }\end{array}$ \\
\hline \hline $\begin{array}{c}\text { A. Patient } \rightarrow \\
\text { (Freq. \%) }\end{array}$ & 81.46 & 12.78 & 5.15 & 0.40 & 0.22 \\
\hline \hline $\begin{array}{c}\text { B. Other Donor } \rightarrow \\
\text { (Freq. \%) }\end{array}$ & 78.00 & 13.69 & 5.96 & 1.94 & 0.42 \\
\hline \hline C. Blood Type $\downarrow$ & & Frequency (\%) \\
\hline O & 48.98 & 49.89 & 38.31 & 62.96 & 48.67 \\
\hline A & 37.18 & 25.28 & 25.06 & 28.78 & 36.00 \\
\hline B & 10.55 & 20.63 & 29.22 & 6.84 & 10.00 \\
\hline \hline AB & 3.29 & 4.19 & 6.41 & 1.43 & 5.33 \\
\hline D. Donor Relation & \multicolumn{5}{|c|}{ Frequency (\%) } \\
\hline Spouse & 34.44 & 40.12 & 43.76 & 32.61 & 41.18 \\
\hline \hline E. PRA Distribution $\downarrow$ & \multicolumn{5}{|c|}{ Frequency (\%) } \\
\hline Low PRA & \multicolumn{5}{|c|}{90.19} \\
\hline Medium PRA & \multicolumn{5}{|c|}{ Frequency (\%) } \\
\hline High PRA & \multicolumn{5}{|c|}{80} \\
\hline \hline F. A2 Subtype Comp. & \multicolumn{5}{|c|}{} \\
\hline F.1. For O Patients & \multicolumn{5}{|c|}{} \\
\hline F.2. For B Patients & \multicolumn{5}{|c|}{} \\
\hline
\end{tabular}

Table 1: Patient and living donor distributions used in simulations: Distribution $\mathrm{A}$ is the frequency of patient races that received live donation, Distribution $\mathrm{C}$ is the blood type frequencies among races of new candidates to the kidney deceased-donor waiting list, and Distribution D is obtained from non-biological donations except kidney exchanges; all three from OPTN data for the year 2014, http://www.optn.org retrieved on 07/10/2015. Distribution $\mathrm{B}$ is 18-60 year-old adult population race distribution in the US and based on 2010 US census retrieved from http://www.census.gov/data.html on 07/10/2015. OPTN/SRTR Annual Report in 2003, for the period 1993-2002, retrieved from http://www . optn.org on 11/22/2004 is used for distribution E. We used a relatively less-recent data report for this distribution, as it was the most detailed data statistic we could find. Distribution F.1 is taken from Nelson et al. (2002) and distribution F.2. is taken from Bryan (2014). The arrow direction (when used) shows the cell direction, along the column or row entries, adding up to frequency 100 percent. For the Bernoulli distributions of D, F.1, and F.2, the alternative event and its probability are omitted for brevity. 
- each medium-PRA patient has a tissue-type incompatibility probability of 45 percent with a random donor, and

- each high-PRA patient has a tissue-type incompatibility probability of 90 percent with a random donor.

After the pool of incompatible patient-donor pairs is determined, these pairs become eligible for exchange. Using the distributions in Table 1, these lead to an average tissue rejection rate of $21.34 \% .^{14}$

We consider seven simulation scenarios differentiated across three dimensions:

1. Under ABO compatibility: This is the baseline scenario and identical to exchange with incompatible pairs.

2. Under subtype A2 compatibility: These six scenarios correspond to the cases in our analytical analysis and are grouped across two dimensions, the timing of blood type A subtype testing and the subtype A2 compatibility technology used:

(a) Timing of the subtyping tests is before joining the kidney-exchange pool: We consider the three subtyping technologies we considered in our theoretical analysis, (i) A2-to-B compatibility, (ii) A2-to-O compatibility, and (iii) full compatibility.

(b) Timing of the subtyping tests is after joining the kidney-exchange pool: We consider the three technologies, (i) A2-to-B compatibility, (ii) A2-to-O compatibility, and (iii) full compatibility.

We consider two different exchange technologies for these 7 simulation scenarios: Optimal 2-way and optimal 2\&3-way exchange. ${ }^{15,16}$

\footnotetext{
${ }^{14}$ This is higher than $11 \%$ rate reported in Zenios, Woodle, and Ross (2001). The data's PRA levels are from the patient population in the US waiting list but not random individuals. Therefore, it could have a bias toward higher PRA individuals. However, we do not consider three-fold reported tissue-incompatibility probability between a female patient and her spouse reported in the literature. This is a mild offset for the higher PRA bias in the data.

${ }^{15}$ For 2-way exchange simulations we use $n=100,500$, and 2000 as the population sizes. Reported t-tests are for $n=2000$, but similar results hold for $n=100$, an $n=500$, as well. On the other hand, because determining a maximal $2 \& 3$-way matching is an NP-complete problem, we use population sizes of $n=100,500$ instead.

${ }^{16}$ In our simulations, to find the maximum number of patients who can benefit from an exchange (for both 2-way and 2\&3-way exchange) we use the CPLEX integer-programming software. We refer the reader to the Appendix of Roth, Sönmez, and Ünver (2007) for the formulation of the optimal exchange problem as an integer program. When compatible pairs are also included in exchange for scenarios 2(b)i-iii, the formulation stands. However, all feasible single-pair cycles are also included in 2-way and 2\&3-way exchange integer program.
} 


\subsection{Simulation Results}

We run $S=500$ 2-way-exchange simulations and report the results as averages and sample standard deviations in Table $2 .{ }^{17}$

Under A2-to-B compatibility when subtyping tests are conducted before joining the pool, the average number of transplants achievable through 2-way exchange (and direct donation if needed) decreases from Scenario 1 - the exchange practice under ABO compatibility only - to Scenario 2(a)i - the de facto exchange policy with A2 compatibility - as predicted by Proposition 1. This decrease is statistically significant with a paired t-test for degrees of freedom 499 with t-statistic 4.3366 that leads to a p-value less than $10^{-5}$.

We also document how the effect of this transition is shared across patients of different ethnic backgrounds (see Table 3). We observe that for whites, there is a decrease, which is statistically significant (with a paired t-test p-value of less than $10^{-5}$ ). For other races, changes are not statistically significant.

The direction of other changes across all treatments are in accordance with the theoretical results of Section 4, as seen in Table 2. In particular, the average number of transplants significantly increases from Scenario 1 and 2(a)i to 2(a)ii and 2(a)iii (cf. Proposition 2), while there is a significant decrease from Scenario 2(a)ii to 2(a)iii (cf. Proposition 3). The same pattern is true for all races (see Table 3). This corresponds to an $11.05-13.74$ percent increase from A2-to-B compatibility to A2-to-O compatibility (from Scenario 2(a)i to 2(a)ii) for different population sizes. Moreover, when the A2 subtype transplantation is done uncoordinated with exchange before the exchange participation decision, even full compatibility brings a substantial decrease to the number of transplants: a 2 percent decrease is observed in the number of transplants with respect to A2-to-O compatibility (from Scenario 2(a)ii to 2(a)iii).

When the subtyping tests are conducted after joining the exchange pool, the corresponding numbers of transplants increase with respect to the cases when subtyping tests are conducted before joining the pool (the signs and statistical significance of differences across scenarios are as predicted by the theoretical results). What is striking is that the maximum average number of transplants under optimal 2-way exchange and live donation (Scenario 2(b)iii), i.e., the maximum achievable, is not too much different (although the increase is highly statistically significant) from the A2-to-O compatibility scenario with subtyping before joining the pool (Scenario 2(a)ii): the difference is only between $0.7-2.5$ percent across different population sizes. If A2-to-B compatibility could totally be eliminated for live donation and we could only use A2-to-O compatibility, even if we could not defer subtype testing until pairs join the exchange pool, we would lose very little with respect to the optimal policy recommendation.

\footnotetext{
${ }^{17}$ In Appendix C, we report the results of simulations for 2\&3-way technology in Tables 5 and 7.
} 


\begin{tabular}{|c|c|c|c|c|c|c|}
\hline \multicolumn{7}{|c|}{ Simulation averages and sample standard deviations of $S=500$ simulations } \\
\hline \multirow{4}{*}{$\begin{array}{c}\text { Incomp. } \\
\text { pairs }\end{array}$} & \multicolumn{6}{|c|}{ 2-way exchanges } \\
\hline & \multirow{3}{*}{$\begin{array}{c}\text { 1. Under } \\
\text { ABO } \\
\text { compatibility }\end{array}$} & \multicolumn{5}{|c|}{ 2. Under A2 compatibility } \\
\hline & & \multirow{2}{*}{$\begin{array}{c}\text { A subtype } \\
\text { test } \\
\text { timing }\end{array}$} & & \multicolumn{3}{|c|}{ A2 compatibility technology } \\
\hline & & & & $\begin{array}{l}\text { i. } \mathrm{A} 2 / \mathrm{A} 2 \mathrm{~B} \rightarrow \mathrm{B} \\
\text { only }\end{array}$ & $\begin{array}{l}\text { ii. } \begin{array}{l}\mathrm{A} 2 \rightarrow \mathrm{O} \\
\text { only }\end{array}\end{array}$ & $\begin{array}{c}\text { iii. } \mathrm{A} 2 / \mathrm{A} 2 \mathrm{~B} \rightarrow \mathrm{B} \\
\text { and } \mathrm{A} 2 \rightarrow \mathrm{O}\end{array}$ \\
\hline \multirow{6}{*}{$\begin{array}{c}49.492 \\
(5.1942) \\
\text { out of } \\
n=100 \\
\text { pairs }\end{array}$} & \multirow{6}{*}{$\begin{array}{c}16.216 \\
(4.9236)\end{array}$} & \multirow{3}{*}{$\begin{array}{c}\text { (a) Before } \\
\text { joining } \\
\text { exchange } \\
\text { pool }\end{array}$} & $\begin{array}{c}\text { Total } \\
\text { transplants }\end{array}$ & $\begin{array}{c}16.638 \\
(5.0666)\end{array}$ & $\begin{array}{c}18.476 \\
(5.4517)\end{array}$ & $\begin{array}{c}18.446 \\
(5.4113)\end{array}$ \\
\hline & & & $\begin{array}{l}\text { B's receiving from own } \\
\text { comp. A2/A2B donors }\end{array}$ & $\begin{array}{c}0.600 \\
(0.5982) \\
\end{array}$ & - & $\begin{array}{c}0.600 \\
(0.5982) \\
\end{array}$ \\
\hline & & & $\begin{array}{l}\text { O's receiving from own } \\
\text { comp. A2 donors }\end{array}$ & - & $\begin{array}{c}0.700 \\
(0.8013)\end{array}$ & $\begin{array}{c}0.700 \\
(0.8013)\end{array}$ \\
\hline & & \multirow{3}{*}{$\begin{array}{l}\text { (b) After } \\
\text { joining } \\
\text { exchange } \\
\text { pool }\end{array}$} & $\begin{array}{c}\text { Total } \\
\text { transplants }\end{array}$ & $\begin{array}{c}16.940 \\
(5.1265)\end{array}$ & $\begin{array}{l}18.554 \\
(5.450)\end{array}$ & $\begin{array}{c}18.944 \\
(5.5352)\end{array}$ \\
\hline & & & $\begin{array}{l}\text { B's receiving from own } \\
\text { comp. A2/A2B donors }\end{array}$ & $\begin{array}{c}0.300 \\
(0.4702)\end{array}$ & - & $\begin{array}{c}0.150 \\
(0.3663)\end{array}$ \\
\hline & & & $\begin{array}{l}\text { O's receiving from own } \\
\text { comp. A2 donors }\end{array}$ & - & $\begin{array}{c}0.500 \\
(0.6882)\end{array}$ & $\begin{array}{c}0.450 \\
(0.6863)\end{array}$ \\
\hline \multirow{6}{*}{$\begin{array}{c}246.430 \\
(12.0187) \\
\text { out of } \\
n=500 \\
\text { pairs }\end{array}$} & \multirow{6}{*}{$\begin{array}{c}90.636 \\
(11.4491)\end{array}$} & \multirow{3}{*}{$\begin{array}{c}\text { (a) Before } \\
\text { joining } \\
\text { exchange } \\
\text { pool }\end{array}$} & $\begin{array}{c}\text { Total } \\
\text { transplants }\end{array}$ & $\begin{array}{c}91.682 \\
(11.8037)\end{array}$ & $\begin{array}{c}103.600 \\
(12.6861)\end{array}$ & $\begin{array}{l}102.0700 \\
(12.5163)\end{array}$ \\
\hline & & & $\begin{array}{l}\text { B's receiving from own } \\
\text { comp. A2/A2B donors }\end{array}$ & $\begin{array}{c}3.014 \\
(1.7527) \\
\end{array}$ & - & $\begin{array}{c}3.014 \\
(1.7527) \\
\end{array}$ \\
\hline & & & $\begin{array}{l}\text { O's receiving from own } \\
\text { comp. A2 donors }\end{array}$ & - & $\begin{array}{c}3.884 \\
(1.954)\end{array}$ & $\begin{array}{c}3.884 \\
(1.954)\end{array}$ \\
\hline & & \multirow{3}{*}{$\begin{array}{l}\text { (b) After } \\
\text { joining } \\
\text { exchange } \\
\text { pool }\end{array}$} & $\begin{array}{c}\text { Total } \\
\text { transplants }\end{array}$ & $\begin{array}{c}93.720 \\
(11.7331)\end{array}$ & $\begin{array}{r}103.952 \\
(12.6679)\end{array}$ & $\begin{array}{c}105.160 \\
(12.7496)\end{array}$ \\
\hline & & & $\begin{array}{l}\text { B's receiving from own } \\
\text { comp. A2/A2B donors }\end{array}$ & $\begin{array}{c}0.596 \\
(0.8958)\end{array}$ & - & $\begin{array}{c}0.252 \\
(0.5487)\end{array}$ \\
\hline & & & $\begin{array}{l}\text { O's receiving from own } \\
\text { comp. A2 donors }\end{array}$ & - & $\begin{array}{c}1.804 \\
(1.2002)\end{array}$ & $\begin{array}{c}1.852 \\
(1.192)\end{array}$ \\
\hline \multirow{6}{*}{$\begin{array}{c}984.800 \\
(23.2186) \\
\text { out of } \\
n=2000 \\
\text { pairs }\end{array}$} & \multirow{6}{*}{$\begin{array}{r}376.700 \\
(22.3124)\end{array}$} & \multirow{3}{*}{$\begin{array}{c}\text { (a) Before } \\
\text { joining } \\
\text { exchange } \\
\text { pool }\end{array}$} & $\begin{array}{c}\text { Total } \\
\text { transplants }\end{array}$ & $\begin{array}{c}374.616 \\
(23.1654)\end{array}$ & $\begin{array}{r}424.852 \\
(25.9950)\end{array}$ & $\begin{array}{c}416.260 \\
(25.4372)\end{array}$ \\
\hline & & & $\begin{array}{l}\text { B's receiving from own } \\
\text { comp. A2/A2B donors }\end{array}$ & $\begin{array}{l}12.180 \\
(3.391)\end{array}$ & - & $\begin{array}{l}12.180 \\
(3.391)\end{array}$ \\
\hline & & & $\begin{array}{c}\text { O's receiving from own } \\
\text { comp. A2 donors }\end{array}$ & - & $\begin{array}{l}15.708 \\
(3.833) \\
\end{array}$ & $\begin{array}{l}15.708 \\
(3.833) \\
\end{array}$ \\
\hline & & \multirow{3}{*}{$\begin{array}{l}\text { (b) After } \\
\text { joining } \\
\text { exchange } \\
\text { pool }\end{array}$} & $\begin{array}{c}\text { Total } \\
\text { transplants }\end{array}$ & $\begin{array}{c}384.238 \\
(23.1129)\end{array}$ & $\begin{array}{c}425.374 \\
(25.9735)\end{array}$ & $\begin{array}{c}428.012 \\
(26.1527)\end{array}$ \\
\hline & & & $\begin{array}{l}\text { B's receiving from own } \\
\text { comp. A2/A2B donors }\end{array}$ & $\begin{array}{c}1.022 \\
(1.4034)\end{array}$ & - & $\begin{array}{c}0.682 \\
(0.7836)\end{array}$ \\
\hline & & & $\begin{array}{l}\text { O's receiving from own } \\
\text { comp. A2 donors }\end{array}$ & - & $\begin{array}{c}3.558 \\
(2.2254)\end{array}$ & $\begin{array}{c}3.882 \\
(3.2902)\end{array}$ \\
\hline
\end{tabular}

Table 2: Simulations for 2-way exchanges (the numbers in parentheses are sample standard deviations, to find the standard errors of the averages, divide the sample standard deviations by $\sqrt{S}=22.36)$. 


\begin{tabular}{|c|c|c|c|c|c|c|c|c|c|}
\hline \multirow{5}{*}{ Races } & \multirow{3}{*}{\multicolumn{2}{|c|}{$\begin{array}{l}\text { Number of } \\
\text { pairs }\end{array}$}} & \multicolumn{7}{|c|}{ Number of patients of each race matched under 2-way exchanges } \\
\hline & & & \multirow{4}{*}{\begin{tabular}{|c|} 
1. Under \\
ABO \\
\end{tabular}} & \multicolumn{6}{|c|}{ 2. Under A2 compatibility } \\
\hline & & & & \multirow{2}{*}{\multicolumn{3}{|c|}{$\begin{array}{l}\text { A subtype test timing: } \\
\text { (a) Before joining exchange pool }\end{array}$}} & \multirow{2}{*}{\multicolumn{3}{|c|}{$\begin{array}{l}\text { A subtype test timing: } \\
\text { (b) After joining exchange pool }\end{array}$}} \\
\hline & Comp. & Inc. & & & & & & & \\
\hline & \multicolumn{2}{|c|}{$\begin{array}{c}\text { (under ABO } \\
\text { compatibility) }\end{array}$} & & $\begin{array}{l}\text { i. } \mathrm{A} 2 / \mathrm{A} 2 \mathrm{~B} \rightarrow 1 \\
\text { only }\end{array}$ & $\begin{array}{l}\text { ii. } \mathrm{A} 2 \rightarrow \mathrm{O} \\
\text { only }\end{array}$ & $\begin{array}{l}\text { iii. } \mathrm{A} 2 / \mathrm{A} 2 \mathrm{~B} \rightarrow \mathrm{B} \\
\text { and } \mathrm{A} 2 \rightarrow \mathrm{O}\end{array}$ & $\begin{array}{l}\text { i. } \mathrm{A} 2 / \mathrm{A} 2 \mathrm{~B} \rightarrow \\
\text { only }\end{array}$ & 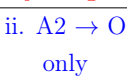 & $\begin{array}{l}\mathrm{A} 2 / \mathrm{A} 2 \mathrm{~B} \rightarrow \mathrm{B} \\
\text { and } \mathrm{A} 2 \rightarrow \mathrm{O}\end{array}$ \\
\hline White & $\begin{array}{r}\mathbf{4 1 . 4 2 8 0} \\
(5.2122)\end{array}$ & $\begin{array}{l}40.0520 \\
(5.0071)\end{array}$ & $\begin{array}{r}\mathbf{1 3 . 0 3 4 0} \\
(4.2869)\end{array}$ & $\begin{array}{r}\mathbf{1 3 . 3 3 6 0} \\
(4.4070)\end{array}$ & $\begin{array}{r}\mathbf{1 4 . 8 5 4 0} \\
(4.7478)\end{array}$ & $\begin{array}{r}\mathbf{1 4 . 8 4 8 0} \\
(4.7360)\end{array}$ & $\begin{array}{r}\mathbf{1 3 . 5 7 6 0} \\
(4.4569)\end{array}$ & $\begin{array}{r}\mathbf{1 4 . 9 3 8 0} \\
(4.7589)\end{array}$ & $\begin{array}{l}\mathbf{1 5 . 2 4 6 0} \\
(4.7958)\end{array}$ \\
\hline Black & $\begin{array}{l}\mathbf{6 . 3 3 8 0} \\
(2.4074)\end{array}$ & $\begin{array}{l}\mathbf{6 . 4 5 6 0} \\
(2.4609)\end{array}$ & $\begin{array}{l}2.0960 \\
(1.4857)\end{array}$ & $\begin{array}{l}\mathbf{2 . 1 8 4 0} \\
(1.5527)\end{array}$ & $\begin{array}{l}2.4100 \\
(1.6270)\end{array}$ & $\begin{array}{l}2.3960 \\
(1.6311)\end{array}$ & $\begin{array}{l}2.2060 \\
(1.5609)\end{array}$ & $\begin{array}{l}\mathbf{2 . 4 1 0 0} \\
(1.6258)\end{array}$ & $\begin{array}{c}2.4720 \\
(1.6718)\end{array}$ \\
\hline Asian & $\begin{array}{l}2.4540 \\
(1.4860)\end{array}$ & $\begin{array}{l}\mathbf{2 . 6 8 0 0} \\
(1.6562)\end{array}$ & $\begin{array}{l}\mathbf{0 . 9 9 4 0} \\
(1.0641)\end{array}$ & $\begin{array}{l}\mathbf{1 . 0 2 6 0} \\
(1.0750)\end{array}$ & $\begin{array}{l}\mathbf{1 . 0 9 6 0} \\
(1.1159)\end{array}$ & $\begin{array}{l}\mathbf{1 . 0 9 0 0} \\
(1.0974)\end{array}$ & $\begin{array}{l}\mathbf{1 . 0 6 4 0} \\
(1.1001)\end{array}$ & $\begin{array}{l}\mathbf{1 . 0 9 0 0} \\
(1.1137)\end{array}$ & $\begin{array}{r}\mathbf{1 . 1 1 8 0} \\
(1.1414)\end{array}$ \\
\hline $\begin{array}{c}\text { American } \\
\text { Indian }\end{array}$ & $\begin{array}{l}\mathbf{0 . 1 8 8 0} \\
(0.4207)\end{array}$ & $\begin{array}{l}\mathbf{0 . 2 1 2 0} \\
(0.4329)\end{array}$ & $\begin{array}{l}\mathbf{0 . 0 6 8 0} \\
(0.2520)\end{array}$ & $\begin{array}{l}\mathbf{0 . 0 7 0 0} \\
(0.2554)\end{array}$ & $\begin{array}{l}\mathbf{0 . 0 8 4 0} \\
(0.2848)\end{array}$ & $\begin{array}{l}\mathbf{0 . 0 8 2 0} \\
(0.2746)\end{array}$ & $\begin{array}{l}\mathbf{0 . 0 7 0 0} \\
(0.2554)\end{array}$ & $\begin{array}{l}\mathbf{0 . 0 8 8 0} \\
(0.2836)\end{array}$ & $\begin{array}{c}\mathbf{0 . 0 8 0 0} \\
(0.2716)\end{array}$ \\
\hline $\begin{array}{l}\text { Pacific } \\
\text { Islander }\end{array}$ & $\begin{array}{l}\mathbf{0 . 1 0 0 0} \\
(0.3003)\end{array}$ & $\begin{array}{l}\mathbf{0 . 0 9 2 0} \\
(0.2962) \\
\end{array}$ & $\begin{array}{l}\mathbf{0 . 0 2 4 0} \\
(0.1532)\end{array}$ & $\begin{array}{l}\mathbf{0 . 0 2 2 0} \\
(0.1468) \\
\end{array}$ & $\begin{array}{l}\mathbf{0 . 0 3 2 0} \\
(0.1762)\end{array}$ & $\begin{array}{l}\mathbf{0 . 0 3 0 0} \\
(0.1708)\end{array}$ & $\begin{array}{l}\mathbf{0 . 0 2 4 0} \\
(0.1532)\end{array}$ & $\begin{array}{l}\mathbf{0 . 0 2 8 0} \\
(0.1651)\end{array}$ & $\begin{array}{r}\mathbf{0 . 0 2 8 0} \\
(0.1651) \\
\end{array}$ \\
\hline $\begin{array}{c}\text { TOTAL } \\
\text { out of } n=100 \text { pairs }\end{array}$ & $\begin{array}{l}\mathbf{5 0 . 5 0 8} \\
(5.1942)\end{array}$ & $\begin{array}{l}49.492 \\
(5.1942)\end{array}$ & $\begin{array}{l}\mathbf{1 6 . 2 1 6} \\
(4.9236)\end{array}$ & $\begin{array}{l}\mathbf{1 6 . 6 3 8} \\
(5.0666)\end{array}$ & $\begin{array}{l}\mathbf{1 8 . 4 7 6} \\
(5.4517)\end{array}$ & $\begin{array}{l}\mathbf{1 8 . 4 4 6} \\
(5.4113)\end{array}$ & $\begin{array}{l}\mathbf{1 6 . 9 4 0} \\
(5.1265)\end{array}$ & $\begin{array}{l}\mathbf{1 8 . 5 5 4} \\
(5.450)\end{array}$ & $\begin{array}{l}\mathbf{1 8 . 9 4 4} \\
(5.5352)\end{array}$ \\
\hline White & $\begin{array}{c}\mathbf{2 0 7 . 8 5 6 0} \\
(11.8609)\end{array}$ & $\begin{array}{c}\mathbf{1 9 8 . 8 3 2 0} \\
(11.3236)\end{array}$ & $\begin{array}{r}\mathbf{7 2 . 1 7 6 0} \\
(9.6702)\end{array}$ & $\begin{array}{l}72.7000 \\
(10.0259)\end{array}$ & $\begin{array}{l}\mathbf{8 2 . 6 2 2 0} \\
(10.9621)\end{array}$ & $\begin{array}{l}\mathbf{8 1 . 3 4 4 0} \\
(10.7693)\end{array}$ & $\begin{array}{l}74.4220 \\
(10.0918)\end{array}$ & $\begin{array}{l}83.0460 \\
(10.8819)\end{array}$ & $\begin{array}{l}83.9060 \\
(10.9744)\end{array}$ \\
\hline Black & $\begin{array}{r}\mathbf{3 1 . 5 3 4 0} \\
(5.4129)\end{array}$ & $\begin{array}{r}32.7300 \\
(5.4398)\end{array}$ & $\begin{array}{r}\mathbf{1 2 . 4 2 8 0} \\
(3.6856)\end{array}$ & $\begin{array}{r}\mathbf{1 2 . 7 6 8 0} \\
(3.6771)\end{array}$ & $\begin{array}{r}\mathbf{1 4 . 2 0 4 0} \\
(3.9595)\end{array}$ & $\begin{array}{r}\mathbf{1 3 . 9 8 8 0} \\
(3.8618)\end{array}$ & $\begin{array}{r}\mathbf{1 2 . 9 2 2 0} \\
(3.7449)\end{array}$ & $\begin{array}{r}14.1240 \\
(3.9288)\end{array}$ & $\begin{array}{l}\mathbf{1 4 . 3 9 4 0} \\
(3.9035)\end{array}$ \\
\hline Asian & $\begin{array}{r}\mathbf{1 2 . 5 5 4 0} \\
(3.3295)\end{array}$ & $\begin{array}{l}\mathbf{1 3 . 2 5 2 0} \\
(3.6572)\end{array}$ & $\begin{array}{l}\mathbf{5 . 4 9 4 0} \\
(2.4236)\end{array}$ & $\begin{array}{l}\mathbf{5 . 6 7 4 0} \\
(2.4641)\end{array}$ & $\begin{array}{l}\mathbf{6 . 1 4 2 0} \\
(2.5516)\end{array}$ & $\begin{array}{l}\mathbf{6 . 1 4 8 0} \\
(2.5907)\end{array}$ & $\begin{array}{l}\mathbf{5 . 8 2 6 0} \\
(2.5394)\end{array}$ & $\begin{array}{l}\mathbf{6 . 1 5 0 0} \\
(2.5052)\end{array}$ & $\begin{array}{l}\mathbf{6 . 2 5 6 0} \\
(2.5713)\end{array}$ \\
\hline $\begin{array}{c}\text { American } \\
\text { Indian }\end{array}$ & $\begin{array}{l}1.0680 \\
(1.0685)\end{array}$ & $\begin{array}{l}1.0200 \\
(1.0206)\end{array}$ & $\begin{array}{l}\mathbf{0 . 3 3 0 0} \\
(0.6244)\end{array}$ & $\begin{array}{l}\mathbf{0 . 3 2 4 0} \\
(0.6097)\end{array}$ & $\begin{array}{l}\mathbf{0 . 3 8 2 0} \\
(0.6730)\end{array}$ & $\begin{array}{l}\mathbf{0 . 3 4 8 0} \\
(0.6290)\end{array}$ & $\begin{array}{l}\mathbf{0 . 3 3 4 0} \\
(0.6255)\end{array}$ & $\begin{array}{l}\mathbf{0 . 3 7 4 0} \\
(0.6442)\end{array}$ & $\begin{array}{l}\mathbf{0 . 3 7 4 0} \\
(0.6220)\end{array}$ \\
\hline $\begin{array}{c}\text { Pacific } \\
\text { Islander }\end{array}$ & $\begin{array}{l}\mathbf{0 . 5 5 8 0} \\
(0.7535)\end{array}$ & $\begin{array}{l}\mathbf{0 . 5 9 6 0} \\
(0.7307) \\
\end{array}$ & $\begin{array}{l}\mathbf{0 . 2 0 8 0} \\
(0.4485) \\
\end{array}$ & $\begin{array}{l}\mathbf{0 . 2 1 6 0} \\
(0.4624) \\
\end{array}$ & $\begin{array}{l}\mathbf{0 . 2 5 0 0} \\
(0.4940) \\
\end{array}$ & $\begin{array}{l}\mathbf{0 . 2 4 2 0} \\
(0.4857) \\
\end{array}$ & $\begin{array}{l}\mathbf{0 . 2 1 6 0} \\
(0.4536)\end{array}$ & $\begin{array}{l}\mathbf{0 . 2 5 8 0} \\
(0.5099)\end{array}$ & $\begin{array}{l}\mathbf{0 . 2 3 0 0} \\
(0.4749) \\
\end{array}$ \\
\hline $\begin{array}{c}\text { TOTAL } \\
\text { out of } n=500 \text { pairs }\end{array}$ & $\begin{array}{l}\mathbf{2 5 3 . 5 7 0} \\
(23.2186)\end{array}$ & $\begin{array}{l}\mathbf{2 4 6 . 4 3 0} \\
(23.2186)\end{array}$ & $\begin{array}{c}\mathbf{9 0 . 6 3 6} \\
(11.4491) \\
\end{array}$ & $\begin{array}{c}\mathbf{9 1 . 6 8 2} \\
(11.8037)\end{array}$ & $\begin{array}{l}\mathbf{1 0 3 . 6 0 0} \\
(12.6861)\end{array}$ & $\begin{array}{c}\mathbf{1 0 2 . 0 7 0 0} \\
(12.5163)\end{array}$ & $\begin{array}{c}93.720 \\
(11.7331)\end{array}$ & $\begin{array}{l}\mathbf{1 0 3 . 9 5 2} \\
(12.6679)\end{array}$ & $\begin{array}{l}\mathbf{1 0 5 . 1 6 0} \\
(12.7496)\end{array}$ \\
\hline White & $\begin{array}{r}833.1060 \\
(23.3396)\end{array}$ & $\begin{array}{c}795.1440 \\
(23.0033)\end{array}$ & $\begin{array}{r}299.6800 \\
(19.3555)\end{array}$ & $\begin{array}{r}\mathbf{2 9 7 . 7 7 8 0} \\
(19.7659)\end{array}$ & $\begin{array}{r}\mathbf{3 3 9 . 3 6 0 0} \\
(22.2173)\end{array}$ & $\begin{array}{r}332.3660 \\
(21.8758)\end{array}$ & $\begin{array}{r}\mathbf{3 0 5 . 9 4 4 0} \\
(19.7554)\end{array}$ & $\begin{array}{r}340.0200 \\
(22.2719)\end{array}$ & $\begin{array}{l}\mathbf{3 4 1 . 9 8 8 0} \\
(22.6046)\end{array}$ \\
\hline Black & $\begin{array}{r}\mathbf{1 2 6 . 3 5 8 0} \\
(10.5689)\end{array}$ & $\begin{array}{c}\mathbf{1 3 0 . 5 1 2 0} \\
(11.1098)\end{array}$ & $\begin{array}{r}\mathbf{5 1 . 5 2 6 0} \\
(7.3608)\end{array}$ & $\begin{array}{r}\mathbf{5 1 . 4 0 8 0} \\
(7.2456)\end{array}$ & $\begin{array}{r}\mathbf{5 7 . 6 6 6 0} \\
(7.4497)\end{array}$ & $\begin{array}{r}\mathbf{5 6 . 5 1 8 0} \\
(7.3210)\end{array}$ & $\begin{array}{r}\mathbf{5 2 . 3 2 4 0} \\
(7.3320)\end{array}$ & $\begin{array}{r}\mathbf{5 7 . 4 2 2 0} \\
(7.7379)\end{array}$ & $\begin{array}{l}\mathbf{5 7 . 9 1 2 0} \\
(7.4857)\end{array}$ \\
\hline Asian & $\begin{array}{r}49.5580 \\
(7.2818)\end{array}$ & $\begin{array}{c}\mathbf{5 3 . 0 2 6 0} \\
(7.1113)\end{array}$ & $\begin{array}{r}\mathbf{2 3 . 3 5 4 0} \\
(5.0095)\end{array}$ & $\begin{array}{r}\mathbf{2 3 . 2 9 0 0} \\
(4.8918)\end{array}$ & $\begin{array}{r}\mathbf{2 5 . 3 7 0 0} \\
(5.1945)\end{array}$ & $\begin{array}{r}\mathbf{2 4 . 9 7 2 0} \\
(5.1886)\end{array}$ & $\begin{array}{r}\mathbf{2 3 . 8 2 0 0} \\
(5.0138)\end{array}$ & $\begin{array}{r}\mathbf{2 5 . 5 3 8 0} \\
(5.2026)\end{array}$ & $\begin{array}{l}\mathbf{2 5 . 6 7 0 0} \\
(5.1160)\end{array}$ \\
\hline $\begin{array}{c}\text { American } \\
\text { Indian }\end{array}$ & $\begin{array}{l}\mathbf{4 . 0 4 2 0} \\
(2.0564)\end{array}$ & $\begin{array}{l}3.9020 \\
(1.9452)\end{array}$ & $\begin{array}{l}\mathbf{1 . 3 0 0 0} \\
(1.1852)\end{array}$ & $\begin{array}{l}\mathbf{1 . 3 0 8 0} \\
(1.1558)\end{array}$ & $\begin{array}{l}\mathbf{1 . 5 1 4 0} \\
(1.2510)\end{array}$ & $\begin{array}{l}\mathbf{1 . 5 0 2 0} \\
(1.2430)\end{array}$ & $\begin{array}{l}\mathbf{1 . 2 9 8 0} \\
(1.1747)\end{array}$ & $\begin{array}{l}\mathbf{1 . 4 3 8 0} \\
(1.2382)\end{array}$ & $\begin{array}{l}\mathbf{1 . 4 9 4 0} \\
(1.2382)\end{array}$ \\
\hline $\begin{array}{c}\text { Pacific } \\
\text { Islander }\end{array}$ & $\begin{array}{l}\mathbf{2 . 1 7 8 0} \\
(1.5083)\end{array}$ & $\begin{array}{l}\mathbf{2 . 1 7 4 0} \\
(1.3621)\end{array}$ & $\begin{array}{l}\mathbf{0 . 8 4 0 0} \\
(0.8740)\end{array}$ & $\begin{array}{l}\mathbf{0 . 8 3 2 0} \\
(0.8997)\end{array}$ & $\begin{array}{l}\mathbf{0 . 9 4 2 0} \\
(0.9531)\end{array}$ & $\begin{array}{l}\mathbf{0 . 9 0 2 0} \\
(0.9392)\end{array}$ & $\begin{array}{l}\mathbf{0 . 8 5 2 0} \\
(0.9032)\end{array}$ & $\begin{array}{l}\mathbf{0 . 9 5 6 0} \\
(0.9633)\end{array}$ & $\begin{array}{l}\mathbf{0 . 9 4 8 0} \\
(0.9397)\end{array}$ \\
\hline $\begin{array}{c}\text { TOTAL } \\
\text { out of } n=2000 \text { pairs }\end{array}$ & $\begin{array}{l}\mathbf{1 0 1 5 . 2 0 0} \\
(23.2186)\end{array}$ & $\begin{array}{l}\mathbf{9 8 4 . 8 0 0} \\
(23.2186)\end{array}$ & $\begin{array}{l}\mathbf{3 7 6 . 7 0 0} \\
(22.3124)\end{array}$ & $\begin{array}{l}\mathbf{3 7 4 . 6 1 6 0} \\
(23.1654)\end{array}$ & $\begin{array}{l}\mathbf{4 2 4 . 8 5 2} \\
(25.9950)\end{array}$ & $\begin{array}{l}\mathbf{4 1 6 . 2 6 0} \\
(25.4372)\end{array}$ & $\begin{array}{l}\mathbf{3 8 4 . 2 3 8} \\
(23.1129)\end{array}$ & $\begin{array}{l}\mathbf{4 2 5 . 3 7 4} \\
(25.9735)\end{array}$ & $\begin{array}{l}\mathbf{4 2 8 . 0 1 2} \\
(26.1527)\end{array}$ \\
\hline
\end{tabular}

Table 3: Simulation averages for 2-way exchanges (Table 2) broken down according to races of the patients. 


\begin{tabular}{|c|c|c|c|c|c|}
\hline \multicolumn{6}{|c|}{ Analytically predicted averages and standard deviations of $S=500$ simulated pools } \\
\hline \multirow{4}{*}{$\begin{array}{l}\text { Incomp. } \\
\text { pairs }\end{array}$} & \multicolumn{5}{|c|}{ 2-way exchanges } \\
\hline & \multirow{3}{*}{$\begin{array}{c}\text { 1. Under } \\
\text { ABO } \\
\text { compatibility }\end{array}$} & \multicolumn{4}{|c|}{ 2. Under A2 compatibility } \\
\hline & & \multirow{2}{*}{$\begin{array}{c}\text { A subtype } \\
\text { test } \\
\text { timing }\end{array}$} & \multicolumn{3}{|c|}{ A2 compatibility technology } \\
\hline & & & $\begin{array}{l}\text { i. } \mathrm{A} 2 / \mathrm{A} 2 \mathrm{~B} \\
\text { only }\end{array}$ & $\begin{array}{l}\text { ii. } \mathrm{A} 2 \rightarrow \mathrm{O} \\
\text { only }\end{array}$ & $\begin{array}{l}\text { iii. } \mathrm{A} 2 / \mathrm{A} 2 \mathrm{~B} \rightarrow \mathrm{B} \\
\text { and } \mathrm{A} 2 \rightarrow \mathrm{O}\end{array}$ \\
\hline $\begin{array}{c}49.420 \\
(5.1942)\end{array}$ & 16.308 & $\begin{array}{l}\text { (a) Before joining } \\
\text { exchange pool }\end{array}$ & $\begin{array}{c}15.906 \\
(4.8514)\end{array}$ & $\begin{array}{c}19.640 \\
(5.3778)\end{array}$ & $\begin{array}{l}19.238 \\
(5.249)\end{array}$ \\
\hline $\begin{array}{c}\text { out of } \\
n=100\end{array}$ & $(4.9778)$ & $\begin{array}{l}\text { (b) After joining } \\
\text { exchange pool }\end{array}$ & $\begin{array}{c}16.406 \\
(4 . .9593)\end{array}$ & $\begin{array}{c}19.898 \\
(5.3551)\end{array}$ & $\begin{array}{c}19.996 \\
(5.3341)\end{array}$ \\
\hline $\begin{array}{c}246.430 \\
(12.0187)\end{array}$ & 90.724 & $\begin{array}{c}\text { (a) Before joining } \\
\text { exchange pool }\end{array}$ & $\begin{array}{c}88.538 \\
(11.3419) \\
\end{array}$ & $\begin{array}{c}107.404 \\
(12.1174)\end{array}$ & $\begin{array}{c}105.218 \\
(12.1174)\end{array}$ \\
\hline $\begin{array}{c}\text { out of } \\
n=500\end{array}$ & $(11.4214)$ & $\begin{array}{l}\text { (b) After joining } \\
\text { exchange pool }\end{array}$ & $\begin{array}{c}91.380 \\
(11.4284)\end{array}$ & $\begin{array}{c}107.904 \\
(12.1926)\end{array}$ & $\begin{array}{c}108.560 \\
(12.2049)\end{array}$ \\
\hline $\begin{array}{c}984.800 \\
(23.2186)\end{array}$ & 376.892 & $\begin{array}{l}\text { (a) Before joining } \\
\text { exchange pool }\end{array}$ & $\begin{array}{c}367.708 \\
(21.9374)\end{array}$ & $\begin{array}{c}43.968 \\
(23.8199)\end{array}$ & $\begin{array}{c}434.784 \\
(23.5528)\end{array}$ \\
\hline $\begin{array}{c}\text { out of } \\
n=2000\end{array}$ & $(22.2724)$ & $\begin{array}{l}\text { (b) After joining } \\
\text { exchange pool }\end{array}$ & $\begin{array}{c}379.120 \\
(22.2172)\end{array}$ & $\begin{array}{c}444.490 \\
(23.8564)\end{array}$ & $\begin{array}{c}446.718 \\
(23.8996)\end{array}$ \\
\hline
\end{tabular}

Table 4: Analytical predictions for 2-way exchanges in simulated pools.

This result also holds across all race groups.

We also compute the analytical predictions stated in our results for our simulated pools for a robustness check of our assumptions. This also helps us to assess the validity of the assumptions made to achieve our analytical results. The corresponding computation averages to the simulation averages are given in Table 4 . We observe that the analytical differences with respect to the baseline scenario 1, ABO compatibility, are slightly above the corresponding simulation differences for A2-to-O compatibility while the reverse is true for A2-to-B compatibilities. ${ }^{18}$ This is caused by two different effects:

- In Assumption UB, we assumed that A2 donors can donate to all O patients. However, this assumption holds on average only 30-40 \% of the time (and we assumed in simulations this rate is $30 \%$, i.e., the worst case scenario, which inflates the difference). Almost half of the A2-O incompatible pairs remain unmatched in the simulations under $\mathrm{A} 2 \rightarrow \mathrm{O}$ technology, while analytical predictions assume that all of these will be matched.

- Assumption TF, in which we assume that there are at least as many A-B pairs as BA pairs holds only on average. Due to the stochastic nature of our simulations, in a minority of the simulated pools, this assumption is violated. On average our assumption is satisfied in $56.2-57 \%$ of the $S=500$ pools randomly generated for different population

\footnotetext{
${ }^{18}$ For example, for $n=100$, the difference between scenarios 2(b)i (A2-to-B) and 1 is 0.098 patients in analytical predictions, while it is 0.724 in the simulation averages. The corresponding numbers for difference between scenarios 2(b)ii (A2-to-O) and 1 are 3.59 and 2.338, respectively.
} 
sizes. Thus, A2/A2B $\rightarrow$ B technology results with slightly less reduction in number of transplants than predicted by analytical results.

These two effects work in opposite directions when both $\mathrm{A} 2 / \mathrm{A} 2 \mathrm{~B} \rightarrow \mathrm{B}$ and $\mathrm{A} 2 \rightarrow \mathrm{O}$ technologies are available. The second effect is smaller than the first effect. Thus, most of our analytical predictions in terms of the directions of the changes remain intact through the simulations.

2\&3-way simulations are very similar, as reported in Tables 5, 7, and 6, respectively, in Appendix C. There is a slight difference in the case of $2 \& 3$-way exchanges: As shown in Appendix B (and the intuition explained in Section 4.3), the efficiency loss due to B-A2 pairs not participating in the exchange is compensated for certain distributions of the pairs in the exchange pool. As a result, the analytically predicted average number of transplants does not decrease when A2-to-B compatibility is integrated (Table 6), and the gap between simulations and analytical results is even smaller compared to only 2-way exchanges (Tables 5 and 6 ).

\section{Conclusion}

Kidney patients of blood types $\mathrm{B}$ and $\mathrm{O}$ are biologically disadvantaged due to a number of factors, and, in the US, they face considerably longer waiting times for transplant kidneys than patients of blood types $\mathrm{A}$ and $\mathrm{AB}$. Both disadvantaged blood types are overrepresented among ethnic minorities, and, in particular, a blood type B patient is significantly more likely to be an ethnic minority than a white. As such, minorities are disproportionately more likely to be biologically disadvantaged for kidney transplantation. Concerns about this inequity contributed to the 2014 reform of the deceased-donor kidney-allocation rule in the US. One of the elements of this recent reform utilizes an advancement in blood-typing technology that enables a certain subtype of blood type A kidneys to be safely transplanted to a fraction of blood type B and blood type $\mathrm{O}$ patients. Under the new rule, an immunologically distinct subset of blood type A kidneys, referred to as subtype A2, are preferentially allocated to blood type B patients with sufficiently low antibody titer levels. Preferential allocation of subtype A2 kidneys is restricted to blood type B patients, in part to give the highest potential benefit to minority patients. This is consistent with the primary goal of the OPTN, as formally stated in the federal Final Rule

The primary goal of the OPTN is to increase and ensure the effectiveness, efficiency, and equity of organ sharing in the national system of organ allocation, and to increase the supply of donated organs available for transplantation.

When restricted to allocation of deceased-donor kidneys, preferential allocation of subtype A2 kidneys is only a distributional policy choice and does not conflict with the OPTN's dual goal of efficiency. 
In this paper, we argue that the specific implementation of this policy in the US de facto extends the preferential allocation to kidney exchange as well. That happens because a 6-month history of sufficiently low antibody anti-A titer value is required for a blood type $\mathrm{B}$ or $\mathrm{O}$ patient to safely receive a subtype A2 transplant. And this history is presently established for blood type B patients so that they can potentially benefit from the preferential allocation system. In contrast, a blood type $\mathrm{O}$ patient typically lacks this required history, thus rendering him ineligible for subtype A2 kidneys in the kidney-exchange pool for at least 6 months. Critically, extending the preferential allocation to kidney exchange may result in a welfare loss in the patient population, not only compared to alternative utilizations of this technology, but even compared to a baseline scenario where the improved subtyping technology is unavailable.

Avoiding the above mentioned welfare loss is not difficult. We promote two adjustments in the current practice to improve the welfare of the patient population. The easier adjustment simply requires systematic establishment of antibody anti-A titer value history for blood type O patients as well as blood type B patients. That simple adjustment breaks the de facto extension of the preferential allocation to kidney exchange. This first adjustment alone makes a significant difference in welfare achievable, although it is not sufficient to obtain full gains.

As a second adjustment, we promote the utilization of the subtyping technology only after blood/tissue-type-incompatible pairs join a kidney-exchange pool. This second adjustment affects the composition of the kidney-exchange pool in a favorable way and results in a further increase in the number of living-donor kidney transplants consistent with the efficiency goal of the OPTN.

The donor blood type A subtyping test is also quite tedious, expensive, and sophisticated. Most doctors are not aware of this practice. Repeated independent testing is needed to verify the A2 subtype, as false positives can happen even under the most sophisticated methods. A false positive can even kill the patient who receives the donor's organ. The health insurance system can potentially play a role in this adjustment by encouraging subtyping tests to be conducted at kidney-exchange programs rather than at hospitals on initial arrival.

Although an A2-subtype-compatible pair cannot be forced to wait for another patient once subtype compatibility is established, the field experience from non-simultaneous altruisticdonor-initiated exchange chains make us optimistic about achievability of this proposal. In such chains, once the patients of incompatible pairs receive a transplant, the donor may wait for months for a suitable patient to be found. Although such donors do not have any obligation for continuing the chain, they often do. This makes us optimistic about the practicality of our proposal. In practice, we also envision that if a certain amount of time is elapsed without a match for such a pair, then this pair should not be discouraged to participate in direct transplantation, either. This will also help with the commitment of pairs for participating in 
exchange after learning their subtype compatibility.

\section{Appendix A Proofs of Section 4}

We start this appendix with a result from Roth, Sönmez, and Ünver (2007) stating the maximum number of transplants when A-subtype compatibility is not considered. This is good to have as a benchmark result before proving further results:

Lemma 1 (Roth, Sönmez, and Ünver, 2007) Suppose LP(i), TF, and UB(i) hold. Consider 2-way exchange under regular ABO compatibility. The maximum number of pairs that can be matched via exchange is

$$
\begin{aligned}
& \#(O-O)_{x}+\#(A-A)_{x}+\#(B-B)_{x}+\#(A B-A B)_{x}+\Gamma+ \\
& 2\left(\#(A-O)_{x}+\#(B-O)_{x}+\#(A B-O)_{x}+\#(A B-A)_{x}+\#(A B-B)_{x}+\#(B-A)\right),
\end{aligned}
$$

where $\Gamma=-\operatorname{odd}_{\#(O-O)_{x}}-\operatorname{odd}_{\#(A-A)_{x}}-\operatorname{odd}_{\#(B-B)_{x}}-\operatorname{odd}_{\#(A B-A B)_{\times}}$.

The intuition behind this result is that:

(i) the incompatible pairs of type $\mathrm{X}-\mathrm{X}$ for each $\mathrm{X}=\mathrm{O}, \mathrm{A}, \mathrm{B}, \mathrm{AB}$ can be matched with each other without using the pairs on the short side, and as a result, none or at most one of them will remain unmatched,

(ii) the pairs on the short side of the market (by $\mathrm{LP}(\mathrm{i})$ and $\mathrm{TF}$ ) are incompatible pairs of types A-O, B-O, AB-O, AB-A, AB-B, and all pairs of type B-A, and hence, they each save one additional pair from the long side and themselves in 2-way exchange. One could be also matched with the remaining single pair of type X-X (as stated above, if one remains), but that would be at the expense of pairs on the long side without changing the number of total transplants.

This intuition for different types of mutual-compatibility graphs was first formalized by Gallai (1963, 1964) and Edmonds (1965), and generalized to situations with compatible pairs joining the exchange pool by Sönmez and Ünver (2014) (as in our scenarios in Section 4.2). We use this result directly or indirectly in our proofs. To state the relevant part of this result for our proofs, first we formalize two additional concepts. We refer to a matching that maximizes the number of patients receiving transplants as a maximal matching. A pair is underdemanded if there exists a maximal matching in which the patient of the pair does not receive a transplant. We state the lemma we use as follows:

Lemma 2 (Gallai-Edmonds decomposition (GED) with possible compatible pairs) (Sönmez and Ünver, 2014) In each maximal matching under 2-way-exchange policy, each pair, 
which is not underdemanded and is mutually compatible with an underdemanded pair, is matched with an underdemanded pair.

We prove our results as follows:

Proof of Proposition 1. (i) Because an A2 donor can donate to a B patient, no tissue-typecompatible B-A2 pair participates in the exchange, and the transplant is via direct donation. This conclusion holds for the tissue-type-compatible B-A2B pairs as well. Thus, the number of transplants via direct donation increases by the number $\#(B-A 2)_{\checkmark}+\#(B-A 2 B)_{\checkmark}$.

(ii) By Assumption UB(i), each B-A pair is compatible with each A-B pair. Moreover, by Assumption TF, each B-A pair is matched with an A-B pair under ABO compatibility. Under A2-to-B compatibility, some of the B-A pairs (tissue-type-compatible B-A2 pairs) do not participate in the exchange and Assumption TF continues to hold with strict inequality for the exchange, which implies that the B-A pairs are on the short side of the exchange and each such pair is matched with an A-B pair. Thus, the number of pairwise exchanges decreases by the number of tissue-type-compatible B-A2 pairs and the number of transplants via exchange decreases by $2 \#(B-A 2)_{\checkmark}$.

Under A2-to-B compatibility, there are possible new matches, which are not possible under ABO compatibility. First, note that each (tissue-type-incompatible) AB-A2B pair can be matched with a B-A1B pair. By Assumption LP(ii), an AB-A2B pair is not underdemanded. By Assumption UB, such a pair is mutually compatible with any B-A1B pair, which is underdemanded by Assumption LP (ii). Moreover, by Assumption UB(i), each AB-A1B pair can be matched with a pair of the same type. Thus, if the number of AB-A1B pairs is even, then no such pair remains unmatched in any maximal matching and the only underdemanded pairs mutually compatible with a AB-A2B pair are B-A1B pairs. This implies that, by Lemma 2, an $\mathrm{AB}-\mathrm{A} 2 \mathrm{~B}$ pair is matched with a B-A1B pair. If the number of AB-A1B pairs is odd, then in a maximal matching, exactly one such pair remains unmatched and an AB-A2B pair can be matched with an AB-A1B pair as well. Because B-A1B pairs are on the long side of the exchange, for each maximal matching where an AB-A2B and an AB-A1B pair are matched, one can obtain another maximal matching where the $\mathrm{AB}-\mathrm{A} 2 \mathrm{~B}$ pair is matched with a $\mathrm{B}-\mathrm{A} 1 \mathrm{~B}$ pair, and an AB-A1B pair remains unmatched. Thus, we can assume without loss of generality that each AB-A2B pair is matched with a B-A1B pair. Because under ABO compatibility, each AB-AB pair is matched only with another AB-AB pair, A2-to-B compatibility increases the number of transplants (via exchange including an AB-AB pair) by the number of (tissuetype-incompatible) AB-A2B pairs. Also, by Assumption UB, each B-A2B pair is compatible with a B-B pair and this implies that each B-B or B-A2B pair can be matched with a B-B or B-A2B pair. Thus, the number of transplants via exchange from ABO compatibility to A2-to-B 
compatibility changes by the following number:

$$
\begin{aligned}
& 2 \#(A B-A 2 B)_{\times}+2\left\lfloor\frac{\#(A B-A 1 B)_{\mathrm{x}}}{2}\right\rfloor+2\left\lfloor\frac{\#(B-A 2 B)_{\mathrm{x}}+\#(B-B)_{\mathrm{x}}}{2}\right\rfloor- \\
& 2\left\lfloor\frac{\#(A B-A B)_{\mathrm{x}}}{2}\right\rfloor-2\left\lfloor\frac{\#(B-B)_{\mathrm{x}}}{2}\right\rfloor-2 \#(B-A 2)_{\triangleleft} .
\end{aligned}
$$

Because $2\left\lfloor\frac{\# T}{2}\right\rfloor=\# T-\operatorname{odd}_{T}$ and $\#(A B-A B)_{\times}=\#(A B-A 1 B)_{\times}+\#(A B-A 2 B)_{\times}$, this number is equal to

$$
\begin{aligned}
& \#(A B-A 2 B)_{\times}+\#(B-A 2 B)_{\times}-2 \#(B-A 2)_{\checkmark}- \\
& \operatorname{odd}_{(A B-A 1 B)_{\times}}-\operatorname{odd}_{(B-A 2 B)_{\times} \cup(B-B)_{\times}}+\operatorname{odd}_{(A B-A B)_{\times}}+\operatorname{odd}_{(B-B)_{\times}}
\end{aligned}
$$

(iii) The result follows from the fact that $\#(B-A 2 B)=\#(B-A 2 B)_{\times}+\#(B-A 2 B)_{\checkmark}$.

Proof of Proposition 2. (i) Because an A2 donor is compatible with an O patient, no tissue-type-compatible O-A2 pair participates in the exchange, and their transplants occur via direct donation.

(ii) By Assumption UB, each incompatible O-A2 pair can be matched with an incompatible OA2 or O-O pair. Because, under ABO compatibility, each O-A pair is on the long side of the exchange, the O-A2 pairs matched with each other and with O-O pairs increase the number of transplants by $\#(O-A 2)_{\times}$and a residual term, depending on whether the total number of incompatible $\mathrm{O}-\mathrm{O}$ and $\mathrm{O}-\mathrm{A} 2$ pairs is odd.

By Assumption LP(iii), each O-A1 pair is underdemanded; the number of O-A1 pairs is greater than the number of $\mathrm{A}-\mathrm{O}$ pairs and the number of incompatible A-A2 pairs. Thus, by Lemma 2, each A-O and A-A2 pair is matched with an O-A1 pair. While incompatible AA pairs (including A-A2 pairs) are matched with each other under ABO compatibility, each incompatible A-A2 pair is matched to an underdemanded pair (in particular, an O-A1 pair) under A2-to-O compatibility. Thus, the number of transplants including an O-A1 pair increases by $\#(A-A 2)_{\times}$and a residual term, depending on whether the number of incompatible A-A1 pairs is odd.

The residual term (due to incompatible O-A2 and A-A2 pairs) depends on whether the cardinalities of certain sets are odd or even. Under ABO compatibility, an O-O pair remains unmatched if the cardinality of the set of incompatible O-O pairs is odd. The same is true for AA pairs. Under A2-to-O compatibility, incompatible A-A1 pairs are matched to each other. Thus, one pair of this type remains unmatched if the cardinality of the set of incompatible AA1 pairs is odd. Similarly, each pair in the set $(O-O)_{\times} \cup(O-A 2)_{\times}$is matched with a pair in the same set. Thus, if the cardinality of this set is odd, then one of these pairs remains unmatched.

(iii) The result follows directly from (i) and (ii). 
Proof of Proposition 4. The number of transplants increases compared to the case where subtype testing is done before joining the exchange pool. The reason is simple: if the test is done before, then some pairs do not participate in the exchange because of subtype compatibility. A compatible B-A2 pair does not participate in the exchange, yet this pair would be matched to an A-B pair if subtype compatibility is ignored, which would increase the number of transplants because A-B pairs are on the long side of the exchange. Thus, this type of loss is prevented by having the test after joining the exchange pool: subtype-compatible pairs on the short side are matched with corresponding pairs on the long side rather than setting them for direct donation. Moreover, if compatible, the B-A2B pairs can be set for direct donation; otherwise, they can be matched with B-A2B pairs or with B-B pairs. Thus, each such pair is matched. The only exception for a direct donation of a compatible B-A2B pair is that if the total number of incompatible B-A2B and B-B pairs is odd, then a compatible B-A2B pair is matched with the remaining pair from the pool of incompatible B-A2B pairs and B-B pairs to prevent any such loss. The number of such 2-way exchanges is constrained not only by the total number of incompatible B-A2B and B-B pairs being odd, but also by the number of compatible B-A2B pairs being positive. This conditional increase in direct donations is captured by the term:

$$
\max \left\{0, \#(B-A 2 B)_{\checkmark}-\operatorname{odd}_{(B-A 2 B)_{\times} \cup(B-B)_{\times}}\right\}
$$

On the other hand, the increase in the number of transplants via exchange due to B-A2B pairs is captured by the following term:

$$
\#(B-A 2 B)_{\times}+\min \left\{\#(B-A 2 B)_{\checkmark}, \operatorname{odd}_{(B-A 2 B)_{\times} \cup(B-B)_{\times}}\right\}+\operatorname{odd}_{(B-B)_{\times}} .
$$

To explain this term, suppose there exists at least one compatible B-A2B pair and the total number of incompatible B-A2B and B-B pairs is odd (otherwise, it is trivial). Under A2-to-B compatibility, all the incompatible B-A2B pairs and B-B pairs are matched, one of them with a compatible B-A2B pair. Thus, the number of transplants via these exchanges is $\#(B-A 2 B)_{\times}+$ $\#(B-B)_{\times}+1$. Because, under ABO compatibility, the number of transplants in exchanges with $\mathrm{B}-\mathrm{B}$ pairs is $\#(B-B)_{\mathrm{x}}-\operatorname{odd}_{(B-B)_{\times}}$, the difference is equivalent to $\#(B-A 2 B)_{\mathrm{x}}+1+$ $\operatorname{odd}_{(B-B)_{\times}}$, the second term standing for $\operatorname{odd}_{(B-A 2 B)_{\times} \cup(B-B)_{\times}}$. Whether there is an unmatched (incompatible) B-A2B or B-B pair depends not only on the total number of such pairs being odd but also on the non-existence of a compatible B-A2B pair. The last item in the residual term in the proposition captures this conditional value.

Also, an AB-A2B pair can be matched with a B-A1B pair, which, by Assumption LP(ii), is on the long side of the exchange. This increases the number of transplants via exchange by $\#(A B-A 2 B)_{\times}$and a residual term depending whether the number of incompatible AB-A1B pairs is odd. 
Proof of Proposition 5. The result is almost the same as the result in Proposition 2, and the reason is that $\mathrm{O}-\mathrm{A}$ pairs are on the long side of the exchange so that direct donation is optimal for compatible O-A2 pairs. The only exception for a direct donation of a compatible O-A2 pair is that if the total number of incompatible O-A2 and O-O pairs is odd, then a compatible O-A2 pair is matched with the remaining pair from the pool of incompatible O-A2 pairs and O-O pairs to prevent any such loss. Clearly, such a 2-way exchange is subject to the existence of at least one compatible O-A2 pair. Note that this is similar to the case under A2-to-B compatibility when a compatible B-A2B pair is matched with the remaining pair from the pool of incompatible B-A2B pairs and B-B pairs. The same argument in Proposition 4 holds here as well, and the term capturing the increase in the number of direct donations is as follows:

$$
\max \left\{0, \#(O-A 2)_{\checkmark}-\operatorname{odd}_{(O-A 2)_{\times} \cup(O-O)_{\times}}\right\} .
$$

Similarly, the increase in the number of transplants via exchange due to O-A2 pairs is given by the following term:

$$
\#(O-A 2)_{\times}+\min \left\{\#(O-A 2)_{\checkmark}, \operatorname{odd}_{(O-A 2)_{\times} \cup(O-O)_{\times}}\right\}+\operatorname{odd}_{(O-O)_{\times}} .
$$

The proof for residual term in the proposition follows from the same argument as in Proposition 4.

\section{Appendix B 2\&3-way Exchanges: Analytical Results}

We analyze the effects of different subtyping technologies on the number of transplants when (not only 2-way exchanges but also) 3-way exchanges are feasible. ${ }^{19}$ Before our analysis, we restate a result from Roth, Sönmez, and Ünver (2007) regarding the number of transplants when no A subtyping technology is available:

Lemma 3 (Roth, Sönmez, and Ünver, 2007) Suppose LP(i), TF, and UB(i) hold. Consider 2-way and 3-way exchange under regular ABO compatibility. The maximum number of pairs that can be matched via exchange is

$$
\begin{aligned}
& \#(A-A)_{x}+\#(B-B)_{x}+\#(O-O)_{x}+\#(A B-A B)_{x}+ \\
& 2\left(\#(A-O)_{x}+\#(B-O)_{x}+\#(A B-O)_{x}+\#(A B-A)_{x}+\#(A B-B)_{x}+\#(B-A)\right)+ \\
& \#(A B-O)_{x}+\min \left\{\#(A-B)-\#(B-A), \#(B-O)_{x}+\#(A B-A)_{x}\right\} .
\end{aligned}
$$

19 The feasibility of 3-way exchanges prevents any potential efficiency loss due to the number of pairs being odd and one pair of this type remaining unmatched. Because there are no remaining pairs for this reason, there is no residual term in each of the following results. We assume for expositional simplicity and without loss of generality that there are at least two pairs of each type in the exchange. 
The intuition behind this result is as follows: This number is different from the number given in Lemma 1 for 2-way exchanges only in the last line and the non-existence of the residual terms (see Footnote 19 for the latter). The last line refers to the fact that:

(i) each AB-O type pair can be matched in a 3-way exchange involving O-A, A-AB (or O-B, B-AB) pairs instead of 2-way (hence, saving 2 additional pairs), and

(ii) the A-B pairs that are not matched with B-A pairs can be saved in 3-way exchanges with B-O and O-A pairs or with AB-A and B-AB pairs.

We extend this rationale to A2 compatibility scenarios by making use of the underdemanded pairs defined for 2-way exchange analysis.

The outline of our analysis in this appendix is as follows: First, we analyze the efficiency implications of blood subtyping technology under the current implementation of blood subtype tests (Appendix B.1). Second, we consider these implications under the change in timing of subtype tests (Appendix B.2).

We define $\Delta$, which we use throughout this appendix, as follows:

$$
\Delta=\#(B-O)_{\mathrm{x}}+\#(A B-A)_{\mathrm{x}}+\#(B-A)-\#(A-B)
$$

\section{B.1 Timing of Subtyping Tests: Before Joining Kidney-Exchange Pool}

Proposition 7 Assume LP(i), LP(ii), TF, UB. Consider the 2-and-3-way-exchange policy. If the compatibility technology changes from ABO compatibility to A2-to- $B$ compatibility, then:

(i) the number of transplants via direct donation increases by $\#(B-A 2)_{\checkmark}+\#(B-A 2 B)_{\checkmark}$,

(ii) the number of transplants via exchange changes by

$$
\begin{aligned}
& \#(A B-A 2 B)_{x}+\#(B-A 2 B)_{x}-2 \#(B-A 2)_{\checkmark}+ \\
& \max \left\{0, \min \left\{\Delta, \#(B-A 2)_{\checkmark}+\#(A-A 2 B)\right\}\right\}, \text { and }
\end{aligned}
$$

(iii) the total number of transplants changes by

$$
\begin{aligned}
& \#(A B-A 2 B)_{x}+\#(B-A 2 B)-\#(B-A 2)_{\checkmark}+ \\
& \max \left\{0, \min \left\{\Delta, \#(B-A 2)_{\checkmark}+\#(A-A 2 B)\right\}\right\}
\end{aligned}
$$

Proof. (i) Given that the subtype test timing is before joining the kidney-exchange pool, the direct donations are selected independently from whether 3-way exchanges are feasible or not. Thus, by part (i) of Proposition 1, the number of transplants via direct donation increases by $\#(B-A 2)_{\checkmark}+\#(B-A 2 B)_{\checkmark}$.

(ii) There are increases in the number of transplants via the following 2-way exchanges: the 
AB-A2B pairs (who are matched with the same type under ABO compatibility) are matched with B-A1B pairs (who are underdemanded by Assumption LP(ii)) and B-A2B pairs (who are on the long side of the exchange under ABO compatibility, but not underdemanded under A2to-B compatibility) are matched with other B-A2B pairs or with B-B pairs. On the other hand, because the compatible B-A2 pairs do not participate in the exchange and the A-B pairs are underdemanded by Assumption TF, there is a decrease in the number of 2-way exchanges by an A-B pair and a B-A pair.

The following 3-way exchanges are possible: a B-O pair, an O-A pair, and an A-B pair (or an $\mathrm{A}-\mathrm{A} 2 \mathrm{~B}$ pair); an $\mathrm{AB}-\mathrm{A}$ pair, an $\mathrm{A}-\mathrm{B}$ pair (or an $\mathrm{A}-\mathrm{A} 2 \mathrm{~B}$ pair), and a $\mathrm{B}-\mathrm{AB}$ pair. In each of these exchanges, two underdemanded pairs (an O-A pair and an A-B pair - or an A-A2B pair - in the first one, and a B-AB pair and an A-B pair - or an A-A2B pair - in the second one) are matched. These 3-way exchanges potentially compensate the efficiency loss due to the compatible B-A2 pairs not participating in the exchange.

Case 1: $\Delta \leq 0$.

Under ABO compatibility, all incompatible B-O and AB-A pairs are matched via 3-way exchanges, including an A-B pair by Lemma 3. This implies that each A-B pair that is not matched with a B-A pair via a 2-way exchange remains unmatched under A2-to-B compatibility. Similarly, no A-A2B pair is matched via a 3-way exchange. Thus, because there are not enough B-O and AB-A pairs to match with the remaining A-B pairs (which are not matched with B-A pairs via a 2-way exchange) via 3-way exchange, switching to the A2-to-B compatibility policy does not increase the number of transplants via 3 -way exchange. ${ }^{20}$ The efficiency loss due to the compatible B-A2 pairs not participating in the exchange cannot be recovered under A2-to-B compatibility.

Case 2: $\Delta>0$.

Under ABO compatibility, each A-B pair, not matched with a B-A pair via a 2-way exchange is matched either with $\mathrm{B}-\mathrm{O}$ pair or an $\mathrm{AB}-\mathrm{A}$ pair via 3-way exchange by Lemma 3. Because $\Delta>0$, the same 3-way exchanges are feasible under A2-to-B compatibility as well. Moreover, there are $\Delta$-many $\mathrm{B}-\mathrm{O}$ and $\mathrm{AB}-\mathrm{A}$ pairs available for further 3 -way exchanges. These B-O and AB-A pairs can be matched with A-B pairs (which are matched with compatible B-A2 pairs

\footnotetext{
${ }^{20}$ Note that under A2-to-B compatibility, a new 3-way exchange is possible: an O-A2B pair, a B-AB pair, and an AB-O pair. Because each AB-O pair is part of a 3-way exchange with two pairs from the long side of the exchange (an O-B pair and a B-AB pair), this new exchange does not increase the number of transplants. Other feasible 3-way exchanges under A2-to-B compatibility are via an A-A2 pair, a B-O pair and an O-A pair, or via an A-A2 pair, an AB-A pair and a B-AB pair. Because an A-A2 pair is not underdemanded and is matched with the same type in each matching, these 3-way exchanges do not increase the number of transplants.
} 
via 2-way exchange under $\mathrm{ABO}$ compatibility but not under A2-to-B compatibility, due to compatible B-A2 pairs not participating in the exchange) or with A-A2B pairs. Thus, the total number of $\mathrm{A}-\mathrm{B}$ pairs and $\mathrm{A}-\mathrm{A} 2 \mathrm{~B}$ pairs available for 3-way exchange with the remaining $\mathrm{B}-\mathrm{O}$ pairs and AB-A pairs is equal to $\#(B-A 2)_{\checkmark}+\#(A-A 2 B)$. Because the number of these new 3 -way exchanges is bounded above by $\Delta>0$, the number of transplants via 3-way exchange increases by $\min \left\{\Delta, \#(B-A 2)_{\checkmark}+\#(A-A 2 B)\right\}$.

(iii) The change in the total number of transplants is equal to the sum of the change in the number of transplants via direct donation and the change in the number of transplants via exchange. Thus, it is equal to the sum of the terms in part (i) and (ii).

Proposition 8 Assume LP(i), LP(iii), TF, UB. Consider the 2-and-3-way-exchange policy. If the compatibility technology changes from ABO compatibility to A2-to-O compatibility, then

(i) the number of transplants via direct donation increases by $\#(O-A 2)_{\checkmark}$,

(ii) the number of transplants via exchange increases by

$$
\begin{aligned}
& \#(O-A 2)_{x}+\#(A-A 2)_{x}+\#(B-A 2)+ \\
& \max \left\{0, \min \left\{\Delta, \#(A B-A 2)_{x}\right\}\right\}, \text { and }
\end{aligned}
$$

(iii) the total number of transplants increases by

$$
\begin{aligned}
& \#(O-A 2)+\#(A-A 2)_{x}+\#(B-A 2)+ \\
& \max \left\{0, \min \left\{\Delta, \#(A B-A 2)_{x}\right\}\right\}
\end{aligned}
$$

Proof. (i) Given that the subtype test timing is before joining the exchange pool, the direct donations are selected independently from whether 3-way exchanges are feasible or not. Thus, by part (i) of Proposition 2, the number of transplants via direct donation increases by \# $(O-$ $A 2)_{\checkmark}$.

(ii) Each tissue-type-incompatible O-A2 pair can be matched with another pair of the same type via 2-way exchange. Similarly, each A-A2 pair is matched with an O-A1 pair, and the remaining A-A pairs (that is, the A-A1 pairs) are matched with each other, such that there is at most one 3-way exchange (only if the cardinality of the set of A-A1 pairs is odd) and there is no unmatched pair in this set. Thus, the total number of transplants via 2-way exchange increases by $\#(O-A 2)_{\mathrm{x}}+\#(A-A 2)_{\mathrm{x}}$.

There are new 3-way exchanges under A2-to-O compatibility, which are not feasible under ABO compatibility. The first one is via pairs of type B-A2, O-A, and A-B. Note that under A2-to-O compatibility, each B-A2 pair joins the exchange pool. Because A-B pairs and OA1 pairs are underdemanded by Assumptions TF and LP(iii), respectively, each B-A2 pair is matched with an O-A1 pair and an A-B pair via a 3-way exchange. Because there are two 
underdemanded pairs in this 3-way exchange (as opposed to only one underdemanded pair (an A-B pair) in the 2-way exchange of A-B and B-A pairs under ABO compatibility), this increases the number of transplants by $\#(B-A 2)$.

Another new 3-way exchange is via the pairs of type AB-A2, O-B and B-AB; or type AB-A2, O-A, and A-AB. Because O-B pairs and B-A1B pairs (also, O-A1 pairs and A-A1B pairs) are underdemanded by Assumptions $\mathrm{LP}(\mathrm{i})$ and $\mathrm{LP}(\mathrm{iii})$, respectively, each AB-A2 pair is matched with an O-B pair and a B-A1B pair (or with an O-A1 pair and an A-A1B pair) via a 3-way exchange. There are two underdemanded pairs (O-B and B-A1B pairs, or O-A1 and A-A1B pairs) in this 3-way exchange (as opposed to only one underdemanded pair (an A-AB pair) in the 2-way exchange of $\mathrm{AB}-\mathrm{A}$ and $\mathrm{A}-\mathrm{AB}$ pairs under $\mathrm{ABO}$ compatibility). Note that, a 2-way exchange with $\mathrm{AB}-\mathrm{A}$ and $\mathrm{A}-\mathrm{AB}$ pairs is not the only exchange where $\mathrm{AB}-\mathrm{A} 2$ pairs are matched under ABO compatibility. Some of the AB-A2 pairs are matched with an A-B pair and a B-AB pair in a 3-way exchange under ABO compatibility. Thus, we need to take the number of these exchanges into account in calculating the increase in the number of transplants via new 3 -way exchanges including an AB-A2 pair.

Case 1: $\Delta \geq \#(A B-A 2)_{\times}$.

Because $\#(A B-A)_{\mathrm{x}}=\#(A B-A 1)_{\mathrm{x}}+\#(A B-A 2)_{\mathrm{x}}$, we have $\#(B-O)_{\mathrm{x}}+\#(A B-A 1)_{\mathrm{x}} \geq$ $\#(A-B)-\#(B-A)$. Each B-A1 pair is matched with an A-B pair via a 2 -way exchange, and each B-A2 pair is matched via a 3-way exchange including an A-B pair (i.e., a 3-way exchange with a B-A2, an O-A1, and an A-B pair). The remaining A-B pairs are matched either with a B-O pair or with an AB-A1 pair via a 3-way exchange. Because there is no remaining A-B pair, each AB-A2 pair can be matched with an O-B pair and a B-AB pair, or with an O-A pair and an A-AB pair, in a 3-way exchange, each of which increases the number of transplants by one compared to ABO compatibility. Thus, the number of transplants increases by $\#(A B-A 2)_{\times}$.

Case 2: $\Delta<\#(A B-A 2)_{\times}$.

First, suppose that $\Delta \leq 0$. Thus, there are not enough B-O and AB-A pairs to match with the remaining A-B pairs (not matched with a B-A pair via a 2-way exchange) via 3-way exchange. By the same argument in Case 1 of Proposition 7, switching to A2-to-O compatibility policy does not increase the number of transplants via 3-way exchanges. Now suppose $\Delta>0$. First, note that $\#(B-O)_{\mathrm{x}}+\#(A B-A 1)_{\mathrm{\times}}<\#(A-B)-\#(B-A)$. Each B-A1 pair is matched with an A-B pair via a 2-way exchange, each B-A2 pair is matched via a 3 -way exchange including an $\mathrm{A}-\mathrm{B}$ pair, and $\#(B-O)_{\mathrm{x}}+\#(A B-A 1)_{\times}$of the remaining $\mathrm{A}-\mathrm{B}$ pairs are matched either with a B-O pair or with an AB-A1 pair via a 3-way exchange. The number of remaining A-B pairs to be matched with AB-A2 pairs via 3-way exchanges is $\#(A-B)-\#(B-A)-\#(B-O)_{\mathrm{x}}-$ 
$\#(A B-A 1)_{\times}$both under ABO compatibility and A2-to-O compatibility. Thus, the number of available AB-A2 pairs for new 3-way exchanges under A2-to-O compatibility (via O-A and $\mathrm{A}-\mathrm{AB}$, or $\mathrm{O}-\mathrm{B}$ and $\mathrm{B}-\mathrm{AB}$ pairs) is ${ }^{21}$

$$
\#(A B-A 2)_{\times}-\left(\#(A-B)-\#(B-A)-\#(B-O)_{\times}-\#(A B-A 1)_{\times}\right) .
$$

Note that this equal to $\Delta$.

Thus, the number of transplants via 3-way exchanges with AB-A2, O-B and B-AB pairs (or AB-A2, O-A and A-AB pairs) increases by the number:

$$
\max \left\{0, \min \left\{\Delta, \#(A B-A 2)_{\times}\right\}\right\}
$$

(iii) The change in the total number of transplants is equal to the sum of the change in the number of transplants via direct donation and the change in the number of transplants via exchange. Thus, it is equal to the sum of the terms in part (i) and (ii).

Proposition 9 Assume $L P(i), L P(i v), T F, U B$. Consider the 2-and-3-way-exchange policy. If the compatibility technology changes from ABO compatibility to full compatibility, then

(i) the number of transplants via direct donation increases by

$$
\#(O-A 2)_{\checkmark}+\#(B-A 2)_{\checkmark}+\#(B-A 2 B)_{\checkmark}
$$

(ii) the number of transplants via exchange changes by

$$
\begin{aligned}
& \#(A B-A 2 B)_{x}+\#(B-A 2 B)_{x}-2 \#(B-A 2)_{\checkmark}+ \\
& \#(O-A 2)_{x}+\#(A-A 2)_{x}+\#(B-A 2)_{x}+ \\
& \max \left\{0, \min \left\{\Delta, \#(B-A 2)_{\checkmark}+\#(A-A 2 B)+\#(A B-A 2)_{x}\right\}\right\}, \text { and }
\end{aligned}
$$

(iii) the total number of transplants changes by

$$
\begin{aligned}
& \#(A B-A 2 B)_{x}+\#(B-A 2 B)+\#(B-A 2)_{x}-\#(B-A 2)_{\triangleleft}+ \\
& \#(O-A 2)+\#(A-A 2)_{x}+ \\
& \max \left\{0, \min \left\{\Delta, \#(B-A 2)_{\triangleleft}+\#(A-A 2 B)+\#(A B-A 2)_{x}\right\}\right\} .
\end{aligned}
$$

\footnotetext{
${ }^{21}$ We assume that under ABO compatibility, an A-B pair, if matched with an AB-A pair in a 3-way exchange, is matched with an AB-A2 pair if and only if all the AB-A1 pairs in the exchange pool are already matched. Without this assumption, this number is the upper bound on the increase in the number of transplants via the 3-way exchanges with AB-A2, O-B and B-AB pairs (or AB-A2, O-A and A-AB pairs).
} 
Proof. (i) The increase in the number of transplants via direct donation is the sum of the terms in part (i) of Propositions 7 and 8. This follows directly from the argument in part (i) of the proofs of these results.

(ii) Parts (3) and (4) follow from part (1) in the expression of Proposition 7(ii) and part (2) in the expression of Proposition 8(ii), respectively. The only difference is that there is $\#(B-A 2)_{\times}$ in part (4), instead of $\#(B-A 2)$ in part (2) in the expression of Proposition 8(ii). The reason is that, because under full compatibility, the compatible B-A2 pairs do not participate in the exchange, only incompatible B-A2 pairs can be matched via a 3 -way exchange including O-A and A-B pairs.

Case 1: $\Delta \geq \#(B-A 2)_{\checkmark}+\#(A-A 2 B)+\#(A B-A 2)_{\mathrm{x}}$.

Because $\#(A B-A)_{\mathrm{x}}=\#(A B-A 1)_{\mathrm{x}}+\#(A B-A 2)_{\mathrm{x}}$, we have $\#(B-O)_{\mathrm{x}}+\#(A B-A 1)_{\mathrm{x}} \geq$ $\#(A-B)-\#(B-A)$. That is, under ABO compatibility, each A-B pair, not matched with a B-A pair via a 2-way exchange, is part of a 3-way exchange including either a $\mathrm{B}-\mathrm{O}$ pair or an AB-A1 pair. Because there are enough B-O and AB-A1 pairs to be matched with these A-B pairs under full compatibility, each AB-A2 pair is matched in a 3-way exchange including an O-B pair and a B-AB pair, or with an O-A pair and an A-AB pair, each of which increases the number of transplants by one compared to ABO compatibility. Moreover, under full compatibility, because \# $(B-A 2)_{\checkmark}$ of B-A pairs do not participate in the exchange, there are \# $(B-A 2)_{\checkmark}$ of A-B pairs not matched with a B-A pair via a 2-way exchange. Similarly, an A-A2B pair can be matched in a 3-way exchange including a B-O pair or an AB-A1 pair. Thus, in total, there are potentially $\#(B-A 2)_{\checkmark}+\#(A-A 2 B)$ pairs which can be matched via 3-way exchange including a B-O pair or an AB-A1 pair. Because $\Delta-\#(A B-A 2)_{\mathrm{x}} \geq \#(B-A 2)_{\checkmark}+\#(A-A 2 B)$, there are enough B-O and AB-A1 pairs to be matched with these pairs in a 3-way exchange. Thus, under full compatibility, each unmatched A-B pair and each A-A2B pair are matched in a 3-way exchange, and each such exchange increases the number of transplants by one. Thus, the number of transplants increases by $\#(B-A 2)_{\checkmark}+\#(A-A 2 B)+\#(A B-A 2)_{\times}$via these 3-way exchanges.

Case 2: $\Delta<\#(B-A 2)_{\checkmark}+\#(A-A 2 B)+\#(A B-A 2)_{\mathrm{x}}$.

Suppose $\Delta<0$. This implies that under ABO compatibility, all B-O and AB-A pairs are matched with A-B pairs via 3-way exchange. Thus, under full compatibility, neither an A-B pair (matched via 2-way exchange under ABO, but not under full compatibility) nor an A-A2B can be matched in a 3-way exchange including one of the B-O and AB-A pairs. Thus, there is no increase in the number of transplants via 3-way exchanges including a B-O pair or an AB-A pair. 
If $0<\Delta$, then under $\mathrm{ABO}$ compatibility each $\mathrm{A}-\mathrm{B}$ pair unmatched via a 2 -way exchange is matched in a 3 -way exchange including a $\mathrm{B}-\mathrm{O}$ pair or an AB-A pair. There are $\#(A-B)-$ $\#(B-A)$ such exchanges. Under full compatibility, each AB-A2 pair can be matched in a 3-way exchange including an O-B pair and a B-AB pair, or an O-A pair and an A-AB pair, and there are potentially $\#(A B-A 2)_{\times}$of these exchanges.

Suppose $0<\Delta<\#(A B-A 2)_{\times}$. By the same argument, as in Case 2 of Proposition 8 , the number of transplants increases by $\Delta$.

Suppose $\Delta>\#(A B-A 2)_{\times}$. Because $\#(B-O)_{\times}+\#(A B-A 1)_{\times}>\#(A-B)-\#(B-A)$, there are $\#(A-B)-\#(B-A)$ 3-way exchanges including an A-B pair. Because each A-B pair in a 3-way exchange is matched with an AB-A1 or a B-O pair under ABO compatibility, ${ }^{22}$ each AB-A2 pair increases the number of transplants by one under full compatibility via 3way exchanges with $\mathrm{O}-\mathrm{B}$ and $\mathrm{B}-\mathrm{AB}$ pairs, or $\mathrm{O}-\mathrm{A} 1$ and $\mathrm{A}-\mathrm{A} 1 \mathrm{~B}$ pairs. Moreover, there are $\left(\#(B-O)_{\mathrm{x}}+\#(A B-A 1)_{\mathrm{x}}\right)-(\#(A-B)-\#(B-A))=\Delta-\#(A B-A 2)_{\mathrm{x}}$ remaining pairs of type B-O or AB-A1 available for 3 -way exchanges with A-B pairs (there are $\#(B-A 2)_{\checkmark}$ such pairs) and A-A2B pairs (there are $\#(A-A 2 B)$ such pairs). Because $\Delta-\#(A B-A 2)_{\times}>0$, there is positive number of these exchanges under full compatibility. Because $\Delta-\#(A B-A 2)_{\times}<$ $\#(B-A 2)_{\checkmark}+\#(A-A 2 B)$, the number of these additional exchanges is $\Delta-\#(A B-A 2)_{\times}$. Thus, under full compatibility, the number of transplants increases by $\Delta$.

(iii) The change in the total number of transplants is equal to the sum of the change in the number of transplants via direct donation and the change in the number of transplants via exchange. Thus, it is equal to the sum of the terms in part (i) and (ii).

\section{B.2 Timing of Subtyping Tests: After Joining Kidney-Exchange Pool}

Proposition 10 Assume LP(i), LP(ii), TF, UB. Consider the 2-and-3-way-exchange policy. If the compatibility technology changes from ABO compatibility to A2-to-B compatibility, then:

(i) the number of transplants via direct donation increases by $\#(B-A 2 B)_{\checkmark}$,

(ii) the number of transplants via exchange increases by

$$
\#(A B-A 2 B)_{x}+\#(B-A 2 B)_{x}+\max \{0, \min \{\Delta, \#(A-A 2 B)\}\}, \text { and }
$$

(iii) the total number of transplants increases by

$$
\#(A B-A 2 B)_{x}+\#(B-A 2 B)+\max \{0, \min \{\Delta, \#(A-A 2 B)\}\}
$$

Proof. The compatible B-A2 pairs are matched via direct donation if the blood subtyping tests are before joining the exchange pool. Because each such direct donation prevents a pairwise

\footnotetext{
${ }^{22}$ See the footnote in Case 2 of the proof of Proposition 8 for an underlying assumption.
} 
exchange with an A-B pair, this results in a decrease in the number of transplants. Thus, given the test timing is after joining the exchange pool, compatible B-A2 pairs are matched with A-B pairs in exchange. Thus, part (1) in the expression of Proposition 7 (ii) does not include the term $-2 \#(B-A 2)_{\checkmark}$. For 3 -way exchanges and the case $\Delta<0$, the same argument as in Case 1 in the proof of Proposition 7 (ii) holds here as well. For the case $\Delta>0$, the only difference is that the total number of A-B pairs and A-A2B pairs available for 3-way exchange with the remaining B-O pairs and AB-A pairs is equal to \# $(A-A 2 B)$ instead of $\#(B-A 2)_{\checkmark}+\#(A-A 2 B)$. Thus, the increase in the number of transplants via 3 -way exchanges with $\mathrm{B}-\mathrm{O}$ and AB-A pairs is equal to the following:

$$
\max \{0, \min \{\Delta, \#(A-A 2 B)\}\} .
$$

Because the other exchanges are not affected by the timing of the tests, the result follows.

Proposition 11 Assume LP(i), LP(iii), UB. Consider the 2-and-3-way-exchange policy. If the compatibility technology changes from ABO compatibility to A2-to-O compatibility, then

(i) the number of transplants via direct donation increases by $\#(O-A 2)_{\checkmark}$,

(ii) the number of transplants via exchange increases by

$$
\#(O-A 2)_{x}+\#(A-A 2)_{x}+\#(B-A 2)+\max \left\{0, \min \left\{\Delta, \#(A B-A 2)_{x}\right\}\right\}, \text { and }
$$

(iii) the total number of transplants increases by

$$
\#(O-A 2)+\#(A-A 2)_{x}+\#(B-A 2)+\max \left\{0, \min \left\{\Delta, \#(A B-A 2)_{x}\right\}\right\},
$$

Proof. The compatible O-A2 pairs are matched via direct donation under A2-to-O compatibility. Moreover, because B-A2 pairs are not compatible under A2-to-O compatibility, these pairs join the exchange pool. Thus, there is no difference between the two scenarios in terms of the timing of blood subtyping tests. Thus, the result is equivalent to the one in Proposition 8.

Proposition 12 Assume LP(i), LP(iv), TF, UB. Consider the 2-and-3-way-exchange policy. If the compatibility technology changes from ABO compatibility to full compatibility, then:

(i) the number of transplants via direct donation increases by $\#(O-A 2)_{\checkmark}+\#(B-A 2 B)_{\checkmark}$,

(ii) the number of transplants via exchange increases by

$$
\begin{aligned}
& \#(O-A 2)_{x}+\#(A-A 2)_{x}+\#(A B-A 2 B)_{x}+\#(B-A 2 B)_{x}+\#(B-A 2)+ \\
& \max \left\{0, \min \left\{\Delta, \#(A B-A 2)_{x}+\#(A-A 2 B)\right\}\right\}, \text { and }
\end{aligned}
$$

(iii) the total number of transplants increases by

$$
\begin{aligned}
& \#(O-A 2)+\#(A-A 2)_{x}+\#(A B-A 2 B)_{x}+\#(B-A 2 B)+\#(B-A 2)+ \\
& \max \left\{0, \min \left\{\Delta, \#(A B-A 2)_{x}+\#(A-A 2 B)\right\}\right\} .
\end{aligned}
$$


Proof. The result follows from Propositions 10 and 11. The difference from Proposition 9 is that compatible B-A2 pairs participate in the exchange as well as incompatible B-A2 pairs, and thus, instead of the term \#(B-A2) $)_{\times}$in expression (4) in part (ii) of Proposition 9, there is the term $\#(B-A 2)$, and the term $\#(B-A 2)_{\checkmark}$ in expression (5) does not appear here.

\section{Appendix C Simulation Results for 2\&3-Way Exchanges}

\begin{tabular}{|c|c|c|c|c|c|c|}
\hline \multicolumn{7}{|c|}{ Averages and standard deviations of $S=500$ simulations } \\
\hline \multirow{4}{*}{$\begin{array}{c}\text { Incomp. } \\
\text { pairs }\end{array}$} & \multicolumn{6}{|c|}{ 2\&3-way exchanges } \\
\hline & \multirow{3}{*}{$\begin{array}{c}\text { 1. Under } \\
\text { ABO } \\
\text { compatibility }\end{array}$} & \multicolumn{5}{|c|}{ 2. Under A2 compatibility } \\
\hline & & \multirow{2}{*}{$\begin{array}{c}\text { A subtype } \\
\text { test } \\
\text { timing } \\
\end{array}$} & & \multicolumn{3}{|c|}{ A2 compatibility technology } \\
\hline & & & & $\begin{array}{l}\text { i. } \mathrm{A} 2 / \mathrm{A} 2 \mathrm{~B} \rightarrow \mathrm{B} \\
\text { only }\end{array}$ & 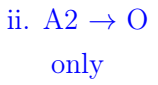 & $\begin{array}{c}\text { iii. } \mathrm{A} 2 / \mathrm{A} 2 \mathrm{~B} \rightarrow \mathrm{B} \\
\text { and } \mathrm{A} 2 \rightarrow \mathrm{O}\end{array}$ \\
\hline \multirow{6}{*}{$\begin{array}{c}49.492 \\
(5.1942) \\
\text { out of } \\
n=100 \\
\text { pairs }\end{array}$} & \multirow{6}{*}{$\begin{array}{c}18.378 \\
(5.2128)\end{array}$} & \multirow{3}{*}{$\begin{array}{c}\text { (a) Before } \\
\text { joining } \\
\text { exchange } \\
\text { pool }\end{array}$} & $\begin{array}{c}\text { Total } \\
\text { transplants }\end{array}$ & $\begin{array}{l}18.692 \\
(5.262)\end{array}$ & $\begin{array}{c}21.740 \\
(5.8447)\end{array}$ & $\begin{array}{c}21.514 \\
(5.8232)\end{array}$ \\
\hline & & & $\begin{array}{l}\text { B's receiving from own } \\
\text { comp. A2/A2B donors }\end{array}$ & $\begin{array}{c}0.550 \\
(0.696)\end{array}$ & - & $\begin{array}{c}0.550 \\
(0.696)\end{array}$ \\
\hline & & & $\begin{array}{l}\text { O's receiving from own } \\
\text { comp. A2 donors }\end{array}$ & - & $\begin{array}{c}0.736 \\
(0.8966)\end{array}$ & $\begin{array}{c}0.736 \\
(0.8966)\end{array}$ \\
\hline & & \multirow{3}{*}{$\begin{array}{c}\text { (b) After } \\
\text { joining } \\
\text { exchange } \\
\text { pool }\end{array}$} & $\begin{array}{c}\text { Total } \\
\text { transplants }\end{array}$ & $\begin{array}{c}18.874 \\
(5.3737)\end{array}$ & $\begin{array}{c}21.750 \\
(5.9352)\end{array}$ & $\begin{array}{c}22.948 \\
(5.9864)\end{array}$ \\
\hline & & & $\begin{array}{l}\text { B's receiving from own } \\
\text { comp. A2/A2B donors }\end{array}$ & $\begin{array}{c}0.116 \\
(0.3617)\end{array}$ & - & $\begin{array}{c}0.060 \\
(0.2693)\end{array}$ \\
\hline & & & $\begin{array}{l}\text { O's receiving from own } \\
\text { comp. A2 donors }\end{array}$ & - & $\begin{array}{c}0.294 \\
(0.5178) \\
\end{array}$ & $\begin{array}{c}0.310 \\
(0.5536) \\
\end{array}$ \\
\hline \multirow{6}{*}{$\begin{array}{c}246.430 \\
(23.2186) \\
\text { out of } \\
n=500 \\
\text { pairs }\end{array}$} & \multirow{6}{*}{$\begin{array}{c}96.956 \\
(11.6453)\end{array}$} & \multirow{3}{*}{$\begin{array}{c}\text { (a) Before } \\
\text { joining } \\
\text { exchange } \\
\text { pool }\end{array}$} & $\begin{array}{c}\text { Total } \\
\text { transplants }\end{array}$ & $\begin{array}{c}97.240 \\
(12.0308)\end{array}$ & $\begin{array}{c}117.292 \\
(12.9746)\end{array}$ & $\begin{array}{c}114.878 \\
(13.1884)\end{array}$ \\
\hline & & & $\begin{array}{l}\text { B's receiving from own } \\
\text { comp. A2/A2B donors }\end{array}$ & $\begin{array}{c}3.014 \\
(1.7437) \\
\end{array}$ & - & $\begin{array}{c}3.014 \\
(1.7437) \\
\end{array}$ \\
\hline & & & $\begin{array}{l}\text { O's receiving from own } \\
\text { comp. A2 donors }\end{array}$ & - & $\begin{array}{c}3.884 \\
(1.9429) \\
\end{array}$ & $\begin{array}{c}3.884 \\
(1.9429) \\
\end{array}$ \\
\hline & & \multirow{3}{*}{$\begin{array}{c}\text { (b) After } \\
\text { joining } \\
\text { exchange } \\
\text { pool }\end{array}$} & $\begin{array}{c}\text { Total } \\
\text { transplants }\end{array}$ & $\begin{array}{c}98.358 \\
(11.6661)\end{array}$ & $\begin{array}{c}117.292 \\
(12.9746)\end{array}$ & $\begin{array}{c}118.596 \\
(13.0767)\end{array}$ \\
\hline & & & $\begin{array}{l}\text { B's receiving from own } \\
\text { comp. A2/A2B donors }\end{array}$ & $\begin{array}{c}0.224 \\
(0.5429)\end{array}$ & - & $\begin{array}{c}0.112 \\
(0.3501)\end{array}$ \\
\hline & & & $\begin{array}{l}\text { O's receiving from own } \\
\text { comp. A2 donors }\end{array}$ & - & $\begin{array}{c}1.404 \\
(1.427)\end{array}$ & $\begin{array}{c}1.456 \\
(1.4656)\end{array}$ \\
\hline
\end{tabular}

Table 5: Simulations for 2\&3-way exchanges. 


\begin{tabular}{|c|c|c|c|c|c|}
\hline \multicolumn{6}{|c|}{ Analytically predicted averages and standard deviations of $S=500$ simulated pools } \\
\hline \multirow{4}{*}{$\begin{array}{l}\text { Incomp. } \\
\text { pairs }\end{array}$} & \multicolumn{5}{|c|}{ 2\&3-way exchanges } \\
\hline & \multirow{3}{*}{$\begin{array}{c}\text { 1. Under } \\
\text { ABO } \\
\text { compatibility }\end{array}$} & \multicolumn{4}{|c|}{ 2. Under A2 compatibility } \\
\hline & & \multirow{2}{*}{$\begin{array}{c}\text { A subtype } \\
\text { test } \\
\text { timing }\end{array}$} & \multicolumn{3}{|c|}{ A2 compatibility technology } \\
\hline & & & $\begin{array}{l}\text { i. } \mathrm{A} 2 / \mathrm{A} 2 \mathrm{~B} \rightarrow \mathrm{B} \\
\text { only }\end{array}$ & 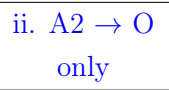 & $\begin{array}{l}\text { iii. } \mathrm{A} 2 / \mathrm{A} 2 \mathrm{~B} \rightarrow \mathrm{B} \\
\text { and } \mathrm{A} 2 \rightarrow \mathrm{O}\end{array}$ \\
\hline $\begin{array}{c}49.420 \\
(5.1942)\end{array}$ & 18.626 & $\begin{array}{c}\text { (a) Before joining } \\
\text { exchange pool }\end{array}$ & $\begin{array}{l}18.688 \\
(5.355) \\
\end{array}$ & $\begin{array}{c}22.696 \\
(5.9352)\end{array}$ & $\begin{array}{c}22.272 \\
(5.7904)\end{array}$ \\
\hline $\begin{array}{c}\text { out of } \\
n=100\end{array}$ & $(5.3234)$ & $\begin{array}{c}\text { (b) After joining } \\
\text { exchange pool }\end{array}$ & $\begin{array}{c}18.878 \\
(5.3737)\end{array}$ & $\begin{array}{c}22.696 \\
(5.9352)\end{array}$ & $\begin{array}{c}22.948 \\
(5.9864)\end{array}$ \\
\hline $\begin{array}{c}246.430 \\
(23.2186)\end{array}$ & 97.152 & $\begin{array}{l}\text { (a) Before joining } \\
\text { exchange pool }\end{array}$ & $\begin{array}{c}97.370 \\
(12.185)\end{array}$ & $\begin{array}{c}117.848 \\
(12.9295)\end{array}$ & $\begin{array}{l}115.322 \\
(13.174)\end{array}$ \\
\hline $\begin{array}{c}\text { out of } \\
n=500\end{array}$ & $(11.6578)$ & $\begin{array}{l}\text { (b) After joining } \\
\text { exchange pool }\end{array}$ & $\begin{array}{c}98.454 \\
(11.8099)\end{array}$ & $\begin{array}{c}117.848 \\
(12.9295)\end{array}$ & $\begin{array}{c}119.146 \\
(13.1071)\end{array}$ \\
\hline
\end{tabular}

Table 6: Analytical predictions for 2\&3-way exchanges in simulated pools.

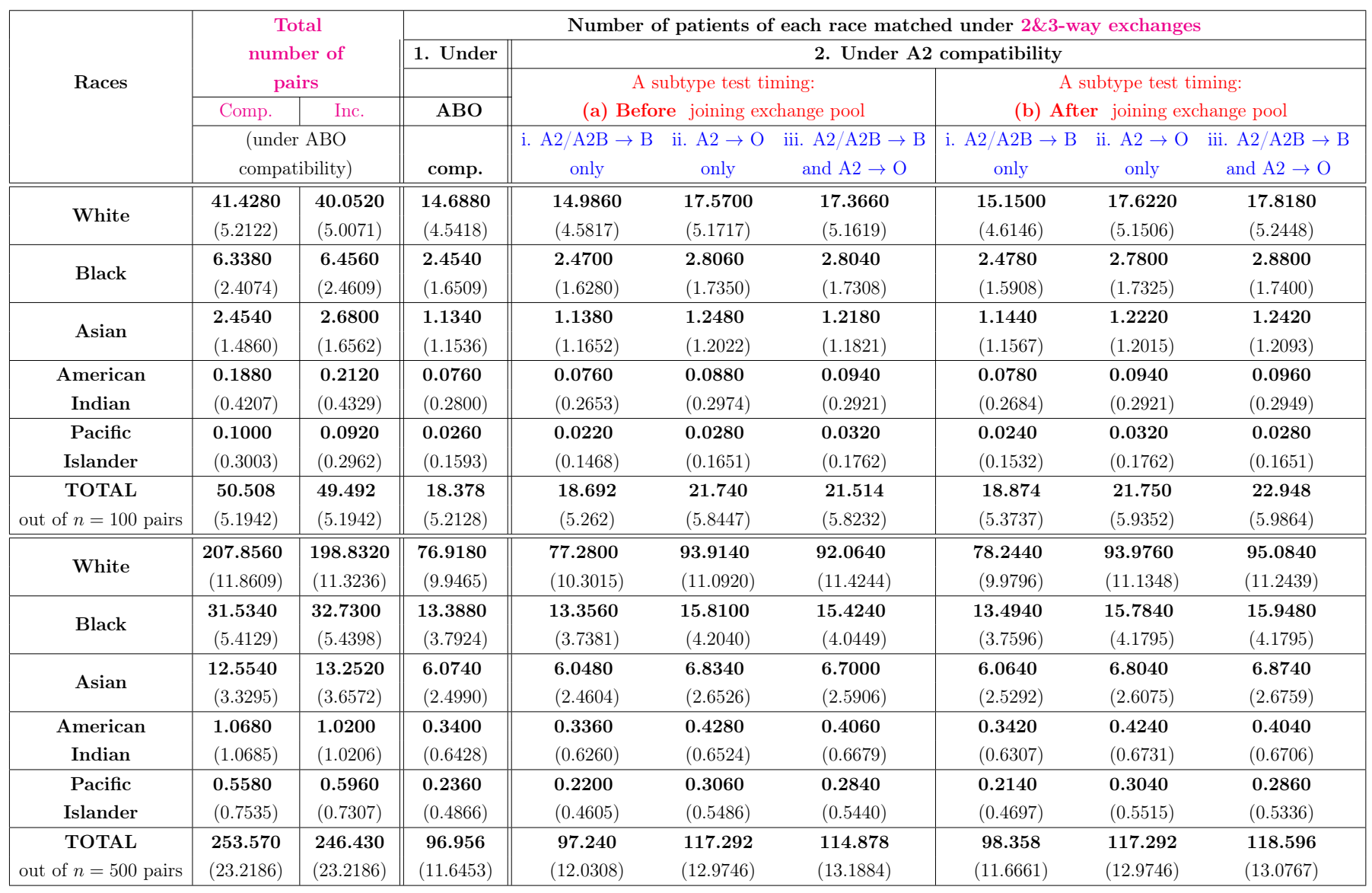

Table 7: Simulations: Averages for 2\&3-way exchanges (Table 5) broken down according to races. 


\section{References}

Akbarpour, Mohammad, Shengwu Li, and Shayan Oveis Gharan (2013), "Thickness and information in dynamic matching markets." Working paper.

Anderson, Ross, Itai Ashlagi, David Gamarnik, and Yash Kanoria (2017), "Efficient dynamic barter exchange." Operations Research, 65, 1446-1459.

Andersson, Tommy and Jörgen Kratz (2016), "Kidney exchange over the blood group barrier." Working paper.

Ashlagi, Itai, David Gamarnik, Michael A. Rees, and Alvin E. Roth (2012), "The need for (long) chains in kidney exchange." Working paper.

Ashlagi, Itai and Alvin E. Roth (2014), "Free riding and participation in large scale, multihospital kidney exchange." Theoretical Economics, 9, 817-865.

Bryan, C. F., H. Polesky, A. B. Eisenbrey, D. A. Sesok-Pizzini, A. M. Luger, D. M. Smith, and B. M. Susskind (2006), "Implications of ABO error rates in proficiency testing for solid organ transplantation." Transplantation, 82(6), 733-736.

Bryan, Christopher F. (2014), "A2/A2B -> B renal transplantation is a component of the new national (UNOS) kidney allocation algorithm." In Heart of America Association of Blood Banks 47th Annual Meeting, volume Spring.

Chun, Youngsub, Eun Jeong Heo, and Sunghoon Hong (2016), "Kidney exchange with immunosuppressants." Working paper.

Dur, Umut, Scott Duke Kominers, Parag A. Pathak, and Tayfun Sönmez (2016), "Reserve design: Unintended consequences and the demise of Boston's walk zones." Journal of Political Economy, forthcoming.

Edmonds, Jack (1965), "Paths, trees, and flowers." Canadian Journal of Mathematics, 17, 449467.

Erdös, Paul and Alfréd Rényi (1960), "The evolution of random graphs." Magyar Tud. Akad. Mat. Kutató Int. Közl., 5, 17-61.

Gallai, Tibor (1963), "Kritische Graphen II." Magyar Tud. Akad. Mat. Kutato Int. Kozl., 8, 373-395. 
Gallai, Tibor (1964), "Maximale Systeme unabhangiger kanten." Magyar Tud. Akad. Mat. Kutato Int. Kozl., 9, 401-413.

Human and Health Services (1998), "Organ Procurement and Transplantation Network." Federal Registrar, 63, 16296-16338.

Massie, A. B., S. E. Gentry, R. A. Montgomery, A. A. Bingaman, and D. L. Segev (2013), "Center-level utilization of kidney paired donation." American Journal of Transplantation, $13,1317-1322$.

Nelson, Paul W. and Christopher F. Bryan (2010), "When will real benefits for minority patients be realized with A2 -> B transplants?" Transplantation, 89, 1310-1311.

Nelson, Paul W., Charles F. Shield, Nicolas A. Muruve, Daniel Murillo, Bradley A. Warady, Mark I. Aeder, and Christopher F. Bryan (2002), "Increased access to transplantation for blood group B cadaveric waiting list candidates by using A2 kidneys: Time for a new national system?" American Journal of Transplantation, 2, 94-99.

Nicolò, Antonio and Carmelo Rodriguez-Álvarez (2017), "Age-based preferences: Incorporating compatible pairs into paired kidney exchange." Games and Economic Behavior, 102, 508524.

Organ Procurement and Transplantation Network (2016), "Policies." January, 11.

Rees, Michael A., Jonathan E. Kopke, Ronald P. Pelletier, Dorry L. Segev, Matthew E. Rutter, Alfredo J. Fabrega, Jeffrey Rogers, Oleh G. Pankewycz, Janet Hiller, Alvin E. Roth, Tuomas Sandholm, M. Utku Ünver, and Robert A. Montgomery (2009), "A non-simultaneous extended altruistic donor chain." New England Journal of Medicine, 360, 1096-1101.

Roth, A. E., T. Sönmez, M. U. Ünver, F. L. Delmonico, and S. L. Saidman (2006), "Utilizing list exchange and non-directed donation through 'chain' paired kidney donations." American Journal of Transplantation, 6, 2694-2705.

Roth, Alvin E., Tayfun Sönmez, and M. Utku Ünver (2004), "Kidney exchange." Quarterly Journal of Economics, 119, 457-488.

Roth, Alvin E., Tayfun Sönmez, and M. Utku Ünver (2005a), "A kidney exchange clearinghouse in New England." American Economic Review Papers and Proceedings, 95, 376-380.

Roth, Alvin E., Tayfun Sönmez, and M. Utku Ünver (2005b), "Pairwise kidney exchange." Journal of Economic Theory, 125, 151-188. 
Roth, Alvin E., Tayfun Sönmez, and M. Utku Ünver (2005c), "Transplant center incentives in kidney exchange." Harvard University and Koç University, unpublished mimeo.

Roth, Alvin E., Tayfun Sönmez, and M. Utku Ünver (2007), "Efficient kidney exchange: Coincidence of wants in markets with compatibility-based preferences." American Economic Review, 97, 828-851.

Sönmez, Tayfun (2013), "Bidding for army career specialties: Improving the ROTC branching mechanism." Journal of Political Economy, 121, 186-219.

Sönmez, Tayfun and M. Utku Ünver (2014), "Altruistically unbalanced kidney exchange." Journal of Economic Theory, 152, 105-129.

Sönmez, Tayfun and M. Utku Ünver (2015), "Enhancing the efficiency of and equity in transplant organ allocation via incentivized exchange." Available as Boston College Working Paper 868.

Sönmez, Tayfun, M. Utku Ünver, and M. Bumin Yenmez (2017), "Incentivized kidney exchange." Working paper.

Terasaki, Paul I., David W. Gjertson, and J. Michael Cecka (1998), "Paired kidney exchange is not a solution to ABO incompatibility." Transplantation, 65.

Toulis, Panos and David C. Parkes (2015), "Design and analysis of multi-hospital kidney exchange mechanisms using random graphs." Games and Economic Behavior, 91, 360 - 382.

Ünver, M. Utku (2010), "Dynamic kidney exchange.” Review of Economic Studies, 77, 372-414.

Zenios, Stefanos A., E. Steve Woodle, and Lainie Friedman Ross (2001), "Primum non nocere: Avoiding increased waiting times for individual racial and blood-type subsets of kidney wait list candidates in a living donor/cadaveric donor exchange program." Transplantation, 72, 648-654. 